Article

\title{
Dual Stimuli-Responsive Copper Nanoparticles Decorated SBA-15: A Highly Efficient Catalyst for the Oxidation of Alcohols in Water
}

\author{
Anju Maria Thomas ${ }^{\mathbb{D}}$, Jerome Peter ${ }^{\mathbb{D}}$, Saravanan Nagappan, Anandhu Mohan and \\ Chang-Sik Ha * \\ Department of Polymer Science and Engineering, Pusan National University, Busan 46241, Korea; \\ anju.05u0@gmail.com (A.M.T.); jestro.12@gmail.com (J.P.); saravananagappa@gmail.com (S.N.); \\ anandhumohangcm@gmail.com (A.M.) \\ * Correspondence: csha@pnu.edu; Tel.: +82-51-510-2407
}

Received: 16 September 2020; Accepted: 13 October 2020; Published: 16 October 2020

\begin{abstract}
In the present work, a temperature and $\mathrm{pH}$-responsive hybrid catalytic system using copolymer-capped mesoporous silica particles with metal nanoparticles is proposed. The poly(2-(dimethylamino)ethyl methacrylate)(DMAEMA)-co-N-tert-butyl acrylamide) (TBA)) shell on mesoporous silica SBA-15 was obtained through free radical polymerization. Then, copper nanoparticles (CuNPs) decorated SBA-15/copolymer hybrid materials were synthesized using the $\mathrm{NaBH}_{4}$ reduction method. SBA-15 was functionalized with trimethoxylsilylpropyl methacrylate (TMSPM) and named TSBA. It was found that the CuNPs were uniformly dispersed in the mesoporous channels of SBA-15, and the hybrid catalyst exhibited excellent catalytic performance for the selective oxidation of different substituted benzyl alcohols in water using $\mathrm{H}_{2} \mathrm{O}_{2}$ as an oxidant at room temperature. The dual (temperature and $\mathrm{pH}-$ ) responsive behaviors of the $\mathrm{CuNPs} / \mathrm{p}(\mathrm{DMAEMA}-\mathrm{co}-\mathrm{TBA}) / \mathrm{TSBA}$ catalyst were investigated using the dynamic light scattering technique. The conversion of catalytic products and selectivity were calculated using gas chromatographic techniques, whereas the molecular structure of the products was identified using ${ }^{1} \mathrm{H}$ and ${ }^{13} \mathrm{C}$ nuclear magnetic resonance (NMR) spectroscopy. The catalyst showed excellent catalytic activity toward the oxidation of alcohol to aldehyde in an aqueous medium below the lower critical solution temperature (LCST) and pKa values (7-7.5) of the copolymer. The main advantages of the hybrid catalyst, as compared to the existing catalysts, are outstanding alcohol conversion (up to $99 \%$ ) for a short reaction time ( $1 \mathrm{~h}$ ), small amount of the catalyst $(5 \mathrm{mg})$, and good recyclability equal to at least five times.
\end{abstract}

Keywords: mesoporous silica; stimuli-responsive polymer; copper nanoparticles; oxidation of alcohols

\section{Introduction}

Selective oxidation of benzyl alcohol to benzaldehyde is essential in perfumery and food industries. The eco-friendly oxidation of benzyl alcohol can be processed using hydrogen peroxide as an oxidant [1-3]. A variety of metal nanoparticle-supported materials have been used as catalysts for the oxidation of benzyl alcohol including supported catalysts such as $\mathrm{Au} / \mathrm{CeO}_{2}$ [4], $\mathrm{Au} / \mathrm{CuO}$ [5], $\mathrm{Pd} /$ hydrotalcite [6], and $\mathrm{Pd} /$ hydroxyapatite [7] nanoparticles. However, precious metals used for the catalysts are usually not so cheap. In this sense, copper-supported catalysts may be an alternative [8-12]. Few works on the use of copper or copper oxide nanoparticles-based catalysts were, however, reported in benzyl alcohol oxidation [8-13]. Moreover, the use of copper nanoparticles (CuNPs) is restricted by Cu's inherent instability under atmospheric conditions, which makes it prone to oxidation. Some supporting materials increase the stability of CuNPs by altering their sensitivity to oxygen, water, and other 
chemical entities, which has encouraged the exploration of alternative Cu-based NPs with more complex structures $[12,14]$.

Mesoporous silica material-supported catalysts have been proven to be the most ideal catalysts because of their high surface area, wall thickness, high porosity, and large and uniform channel size. To prevent the agglomeration of CuNPs, the use of a stabilizing agent is essential for the synthesis of CuNPs [15-17]. Although the wet impregnation method is an effective method for incorporating metals on a silica surface, that method usually results in non-uniform dispersion. This becomes particularly troublesome because in those mesoporous supports, pores can be completely blocked, reducing the active surface areas. To ensure better incorporation of metal nanoparticles, functional groups are often decorated onto the mesoporous silica surface through a surface modification process [17-19]. The grafted functional groups such as amine, thiol, cyano, vinyl, alkyl, and phenyl can act as anchors to enhance the interaction between the silica wall and the metal precursor [20-23]. Here, we used trimethoxy silyl propyl methacrylate (TMSPM) for the surface functionalization of SBA-15. Then, we have covered the surface of SBA-15 with poly(N,N-dimethylaminoethyl methacrylate) ( $\mathrm{p}$ (DMAEMA)), which is an example of a thermo-responsive polymer and it also possesses $\mathrm{pH}$ sensitivity. The lower critical solution temperature (LCST) of $\mathrm{p}$ (DMAEMA) is dependent on the $\mathrm{pH}$. At high $\mathrm{pH}$, the amino groups are protonated and the electrostatic repulsions between the polymeric chains impede the collapse [24-26]. One of the interesting research areas for stimuli-responsive materials is to tune the phase transition temperatures near the ambient region $\left(0-100^{\circ} \mathrm{C}\right)$. The LCST will be lowered when the polymer is copolymerized with a hydrophobic co-monomer, while the LCST will be increased by adding a hydrophilic co-monomer. Herein, we used tert-butyl acrylamide (TBA) as a co-monomer with PDMAEMA to lower the LCST value. The LCST of DMAEMA is in the range of $45-50{ }^{\circ} \mathrm{C}$. After copolymerization with TBA, LCST reaches approximately $20-25^{\circ} \mathrm{C}$. The thermo-responsive behavior of the catalyst can be explained by the polymer covering the surface of mesoporous silicas, which act as a gate to the pore entrance $[27,28]$. Below the LCST, the polymer is swollen, so the gate will open, and the reactant molecules can easily penetrate the pores and react with the metal nanoparticles inside the pores. Above the LCST, the polymer layer on the SBA-15 surface was shrunken, so the gate was closed, and it was difficult for the reactants to approach the metal side. Polymer-grafted mesoporous silicas can be used in the field of drug delivery, catalysis, and separation, particularly for the oxidation of various alcohols. In this study, we synthesized an organic-inorganic nanohybrid catalyst based on copper nanoparticles-decorated copolymer-grafted mesoporous SBA-15 silica material. This study aims to investigate the effect of the dual stimuli (temperature and $\mathrm{pH}$ )-responsive behavior of the catalyst used for the oxidation of benzyl alcohol in a water medium using $\mathrm{H}_{2} \mathrm{O}_{2}$ as an oxidant. We also tested the reusability of the catalyst.

\section{Materials and Methods}

\subsection{Synthesis of $p(D M A E M A-c o-T B A) / T S B A$ Hybrid Particles}

The copolymerization reaction was performed in a three-neck flask equipped with a reflux condenser to synthesize p(DMAEMA-co-TBA). During the experiment, the reaction was homogenized using a magnetic stirrer. A solution of $0.6 \mathrm{~g}$ of DMAEMA and $0.4 \mathrm{~g}$ of TBA was placed in a round bottom flask. Ethanol and water mixtures were used as solvents under nitrogen flow. Then, $0.2 \mathrm{~g}$ of modified SBA-15 (named TSBA, which is SBA-15 functionalized with trimethoxyl silyl propyl methacrylate (TMSPM); the detailed experimental procedure is provided in the Supplementary Data) was added to the reaction mixture. After $1 \mathrm{~h}, 0.027 \mathrm{~g}$ of potassium persulfate $\left(\mathrm{K}_{2} \mathrm{~S}_{2} \mathrm{O}_{8}\right)$ initiator was added. The mixture was heated to $80^{\circ} \mathrm{C}$, and the polymerization continued for $7 \mathrm{~h}$, followed by centrifugation at 10,000 rpm and washed thoroughly with water and ethanol. The mixture was then dried at $50{ }^{\circ} \mathrm{C}$ under a vacuum to obtain the product denoted as p(DMAEMA-co-TBA)/TSBA. 


\subsection{Loading of Copper Nanoparticles (CuNPs) into p(DMAEMA-co-TBA)/TSBA}

$0.05 \mathrm{~g}$ of poly(DMAEMA-co-TBA)/TSBA was dispersed in water after sonication for $15 \mathrm{~min}$. The suspension was continuously stirred for $2 \mathrm{~h}$ at room temperature to achieve a complete dispersion of SBA-15. Then, a $0.01 \mathrm{M}$ solution of $\mathrm{Cu}(\mathrm{II}) \mathrm{Br}$ was added, and the reaction was stirred for $6 \mathrm{~h}$ under a nitrogen atmosphere. Finally, the metal precursor was reduced to metal nanoparticles through reduction with the addition of $0.1 \mathrm{M} \mathrm{NaBH}_{4}$ solution under mild stirring. The original clear white solution turned black after the reaction. The reaction was stirred for $1 \mathrm{~h}$. Then, the catalyst was retrieved through filtration, washed carefully with water, and dried at $50{ }^{\circ} \mathrm{C}$. The obtained product was named CuNPs/p(DMAEMA-co-TBA)/TSBA. Chemicals for the synthesis of materials in this Section 2 and their basic characterization methods are described in the Supplementary Materials.

\subsection{Catalysis-Oxidation of Alcohols}

The synthesized CuNPs-supported hybrid catalyst was tested for the oxidation of alcohols using benzyl alcohol as a model substrate and optimized using various parameters such as the oxidant, solvent, and temperature. In a typical experiment, $5 \mathrm{mg}$ of the catalyst was dispersed in $5 \mathrm{~mL}$ of deionized water and stirred for $10 \mathrm{~min}$. Then, benzyl alcohol $(1 \mathrm{mmol})$ was added, and nitrogen purging was conducted for $2 \mathrm{~min}$, followed by the dropwise addition of hydrogen peroxide ( $1 \mathrm{mmol})$. After the addition of $\mathrm{H}_{2} \mathrm{O}_{2}$ the $\mathrm{pH}$ of the system was found to be 6 . The reaction mixture was stirred at an ambient temperature. After the reaction was completed, the mixture was filtered, separated, and extracted using diethyl ether $(2 \times 10 \mathrm{~mL})$. The catalytic products were analyzed using gas chromatography (GC). Similar reaction conditions and methodology were used for other alcoholic substrates, and the conversion and selectivity were estimated. The resulting products were further purified using column chromatography, and their molecular structures were identified by ${ }^{1} \mathrm{H}$ and ${ }^{13} \mathrm{C}$ nuclear magnetic resonance (NMR) spectra using $\mathrm{CDCl}_{3}$ solvent.

\section{Results and Discussion}

\subsection{Characterization of Materials}

The synthesis routes for the grafting of the copolymer on the SBA-15 particles are shown in Figure 1. In the first step, the external surface of SBA-15 was functionalized with TMSPM (Figure 1a). In the second step, copolymerization of the grafted particles with DMAEMA and TBA was conducted (Figure 1b). The structure of the copolymer and its grafting procedure are shown in Figure 1b. Finally, the CuNPs were loaded into the p(DMAEMA-co-TBA)/TSBA (Figure 1c). The temperature- and pH-responsive behavior of the catalyst depends upon the shrinking/swelling and protonation/deprotonation environment of the dimethyl amino groups (pKa of 7-7.5) of the copolymer chain upon heating/cooling. At low $\mathrm{pH}$ levels, the amino groups in DMAEMA have a positive charge; therefore, maximum swelling occurs due to the repulsion of like charges generated in the polymer, and the pores are found to be in the relaxed open state. In contrast, the closing of the windows of mesopores occurs at high $\mathrm{pH}$ conditions due to the deprotonation of amino groups (Figure 1d).

Figure 2 illustrates the Fourier transform infrared (FT-IR) spectra of SBA-15, TSBA, and $\mathrm{p}$ (DMAEMA-co-TBA)/TSBA. The detailed band assignment of SBA-15 and functionalized mesoporous silica has been reported in previous literatures [26,27]. The broad bands of adsorption at approximately $3437 \mathrm{~cm}^{-1}$ can be assigned to the water molecules hydrogen-bonded to each other and to $\mathrm{Si}-\mathrm{OH}$ groups. The bands at approximately $1636 \mathrm{~cm}^{-1}$ are due to the bending vibrations of $\mathrm{O}-\mathrm{H}$ bonds in $\mathrm{H}_{2} \mathrm{O}$ groups, overlapping with stretching vibrations of $\mathrm{C}-\mathrm{O}-\mathrm{C}$. The bands near $1082 \mathrm{~cm}^{-1}$ correspond to the $\mathrm{Si}-\mathrm{O}-\mathrm{Si}$ asymmetric stretching vibrations, overlapped with $\mathrm{Si}-\mathrm{O}-\mathrm{C}, \mathrm{C}-\mathrm{O}-\mathrm{C}$, and $\mathrm{Si}-\mathrm{C}$ vibrations. The bands at $962 \mathrm{~cm}^{-1}$ are attributed to the stretching vibrations of free $\mathrm{Si}-\mathrm{OH}$ groups on the amorphous solid sample surface, while $\mathrm{CO}$ stretching vibration bonds are also overlapped in the range. The characteristic peak at $1701 \mathrm{~cm}^{-1}$ for the modified SBA- 15 could be assigned to $C=O$ stretching, and the peaks at $2916 \mathrm{~cm}^{-1}$ and $2848 \mathrm{~cm}^{-1}$ correspond to the $\mathrm{C}-\mathrm{H}$ stretching and bending vibration, respectively, present in TMSPM. 
The sharp band at $1728 \mathrm{~cm}^{-1}$ suggests the existence of the $\mathrm{C}=\mathrm{O}$ group in DMAEMA. However, there is a broadening of the band intensity at $3437 \mathrm{~cm}^{-1}$ because of the presence of $\mathrm{NH}$ and $\mathrm{OH}$ functional groups, indicating the successful attachment of TBA and DMAEMA to the surface of SBA-15.

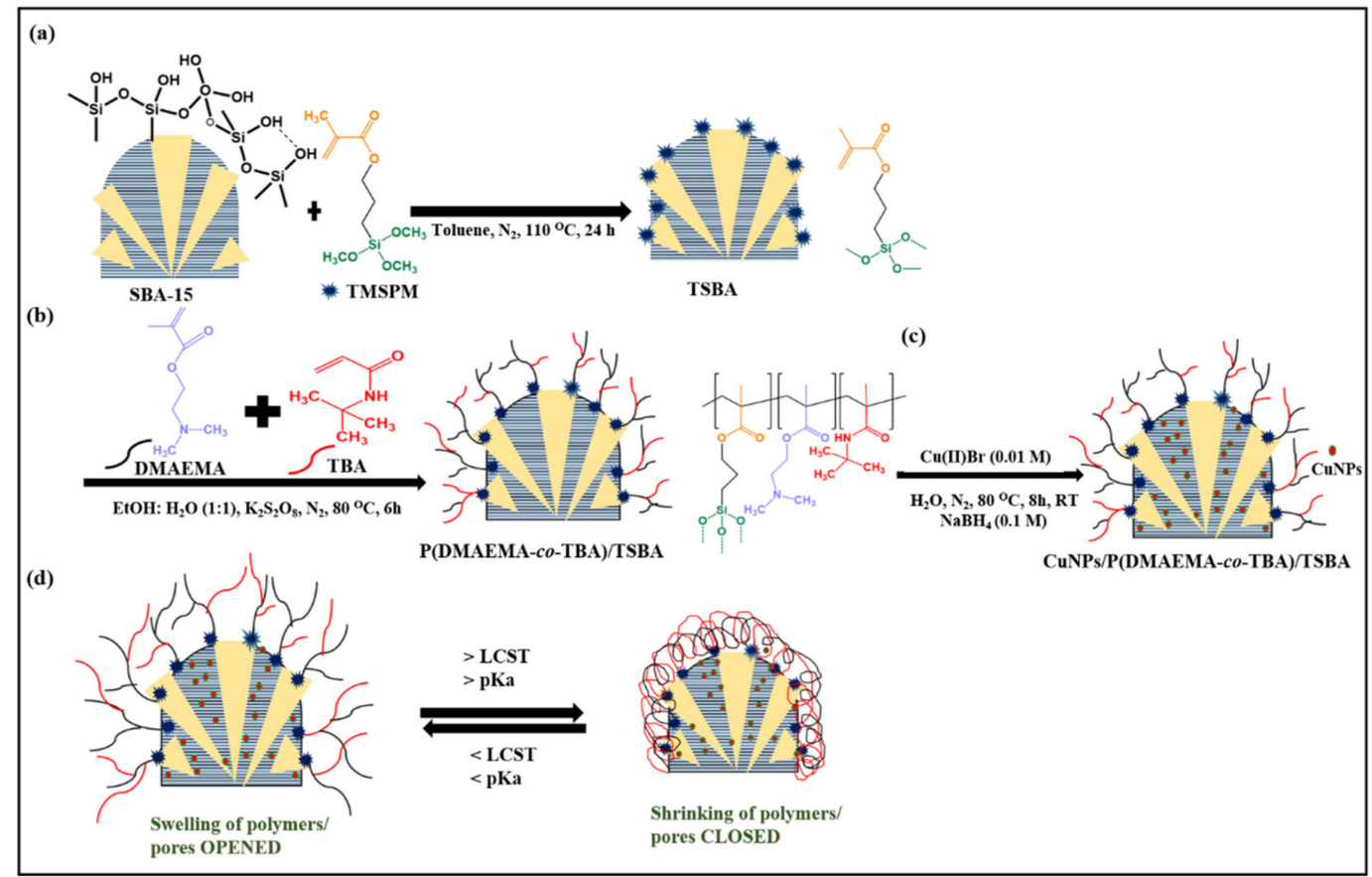

Figure 1. (a) Modification of surface hydroxyl groups on the SBA-15 with silane group of trimethoxy silyl propyl methacrylate (TMSPM); (b) structure of the copolymer grafted surface of SBA-15; (c) incorporation of copper nanoparticles (CuNPs) on to the p(DAMEAM-co-TBA)/TSBA (where, TSBA is the modified SBA-15); (d) closing of opening of mesopores with respect to the responsive behavior of the copolymer. Chemicals for the syntheses of materials in Section 2 and their basic characterization methods are described in the Supplementary Materials.

Field emission scanning electron microscopy (FESEM) images of rod-shaped SBA-15, $\mathrm{p}$ (DMAEMA-co-TBA)/TSBA, and CuNPs/p(DMAEMA-co-TBA)/TSBA are shown in Figure 3. SBA-15 shows long rod-shaped particles with lengths and widths of $1 \mu \mathrm{m}$ and $130 \mathrm{~nm}$, respectively, as reported in previous literature [26] (Figure 3a). The surface of SBA-15 was quite smooth and clear, without any impurities. The interaction between the surfactant and the precursor is responsible for the formation of mesoporous materials during the self-assembly process. The exterior surface became rougher after polymer coating, which confirms the covering of SBA-15 with the copolymer. (Figure 3b). A small amount of CuNP loading does not affect the morphology of the mesoporous silica. The CuNPs/p(DMAEMA-co-TBA)/TSBA also displayed SBA-15 morphology (Figure 3c). The small particles observed on the surface confirmed the presence of agglomerated CuNPs.

Figure 4 displays high-resolution transmission electron microscopy (HRTEM) images of SBA-15, p(DMAEMA-co-TBA)/TSBA, and CuNPs/p(DMAEMA-co-TBA)/TSB. The images clearly show the well-ordered arrangement of mesopores in SBA-15 for all three samples. The mesoporous structure was maintained even after modification, which confirms that the modification does not affect the pore structure of SBA-15. After polymerization with DMAEMA and TBA, the material preserved the mesoporosity (Figure $4 \mathrm{~b}$ ). These images further support the structural integrity of the silicate matrix, which was maintained even after metal incorporation. The presence of dark spots confirms the presence of CuNPs both inside and outside of the mesopores (Figure 4c). HRTEM images, together with energy dispersive X-ray (EDX) mapping, indicate the presence of CuNPs in the CuNPs/p(DMAEMA-co-TBA)/TSBA catalyst. The weight \% of the loaded CuNPs was found to be 
$1.02 \%$ in the catalyst (Figure 5). Moreover, the presence of other elements, such as $\mathrm{Si}, \mathrm{C}$, and O, was also confirmed.

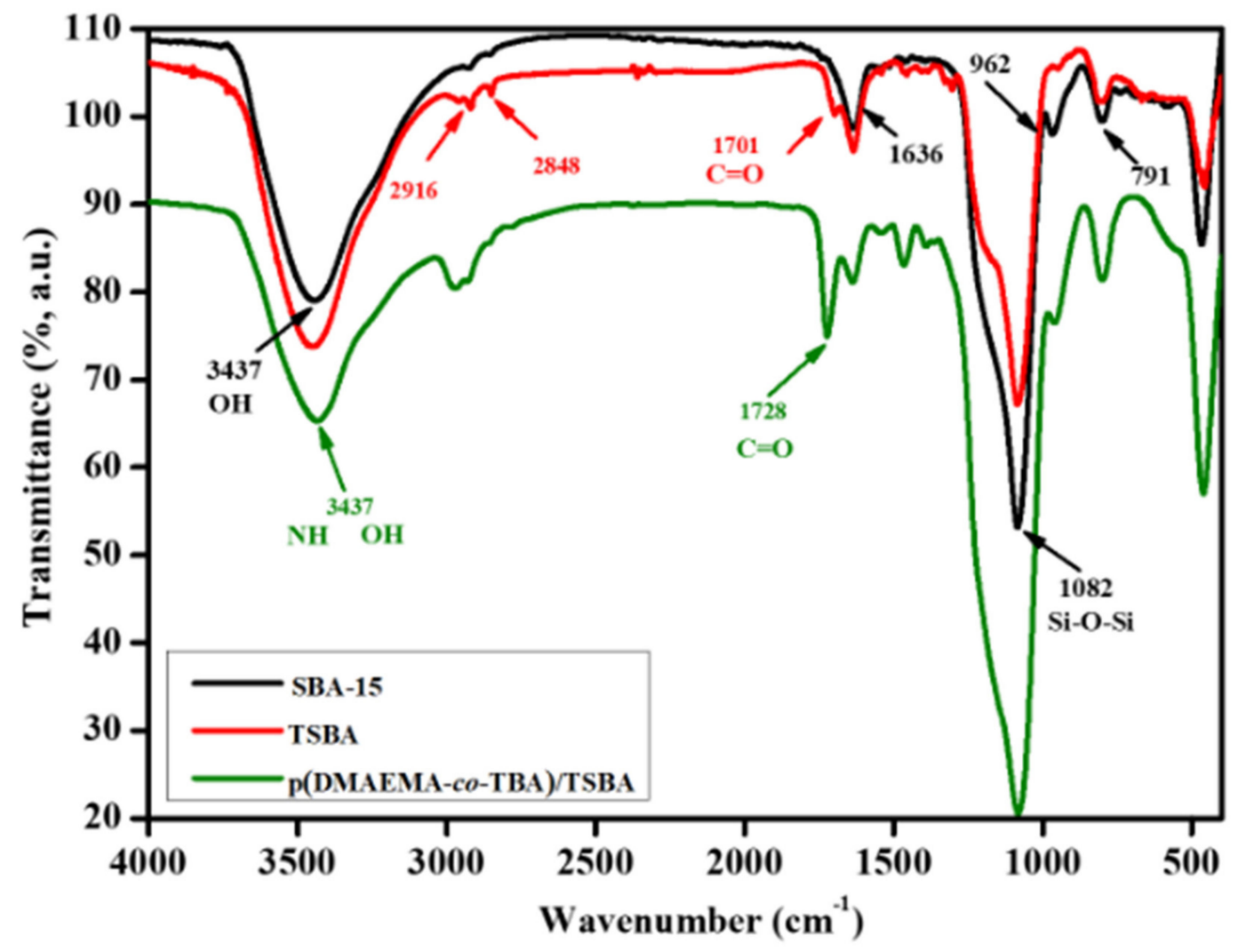

Figure 2. FT-IR spectra of SBA-15, TSBA, and p(DMAEMA-co-TBA)/TSBA.
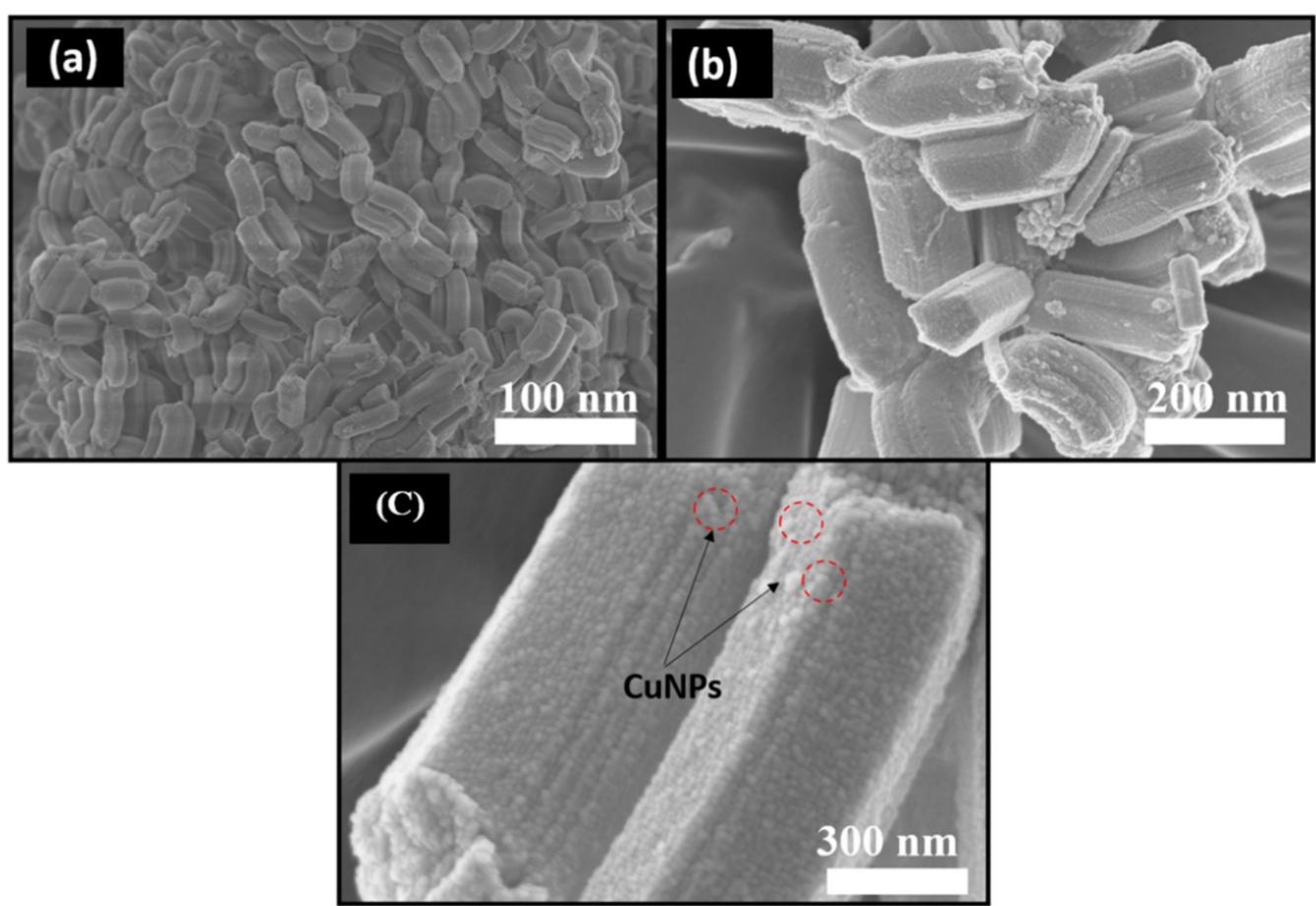

Figure 3. Field emission scanning electron microscopy (FESEM) images of (a) SBA-15; (b) $\mathrm{p}$ (DMAEMA-co-TBA)/TSBA; (c) CuNPs/p(DMAEMA-co-TBA)/TSBA. 


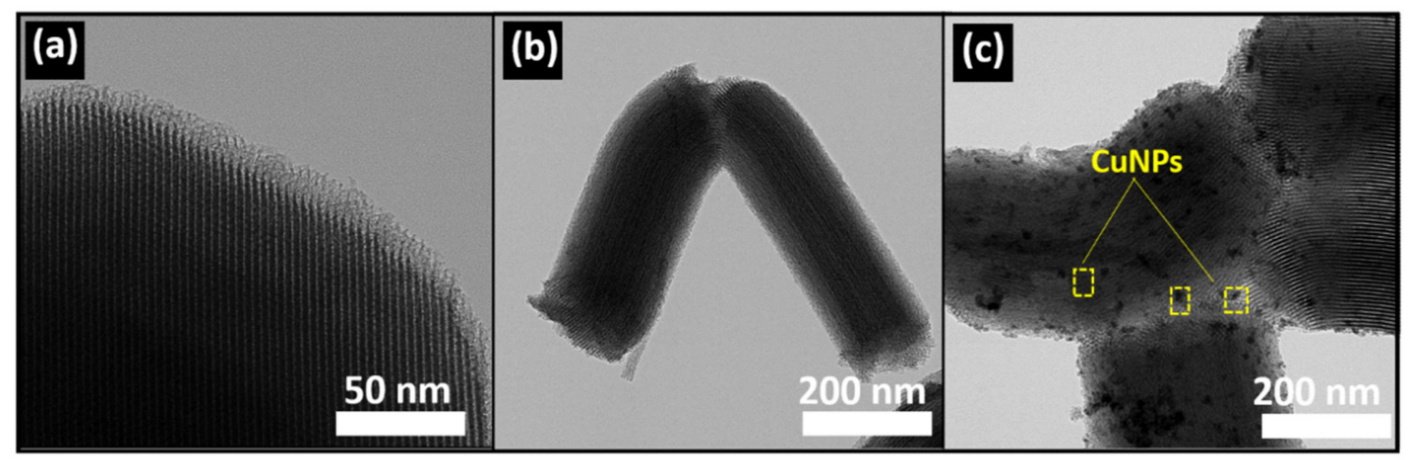

Figure 4. High-resolution transmission electron microscopy (HRTEM) images of (a) SBA-15; (b) p(DMAEMA-co-TBA)/TSBA, and (c) CuNPs/p(DMAEMA-co-TBA)/TSBA.

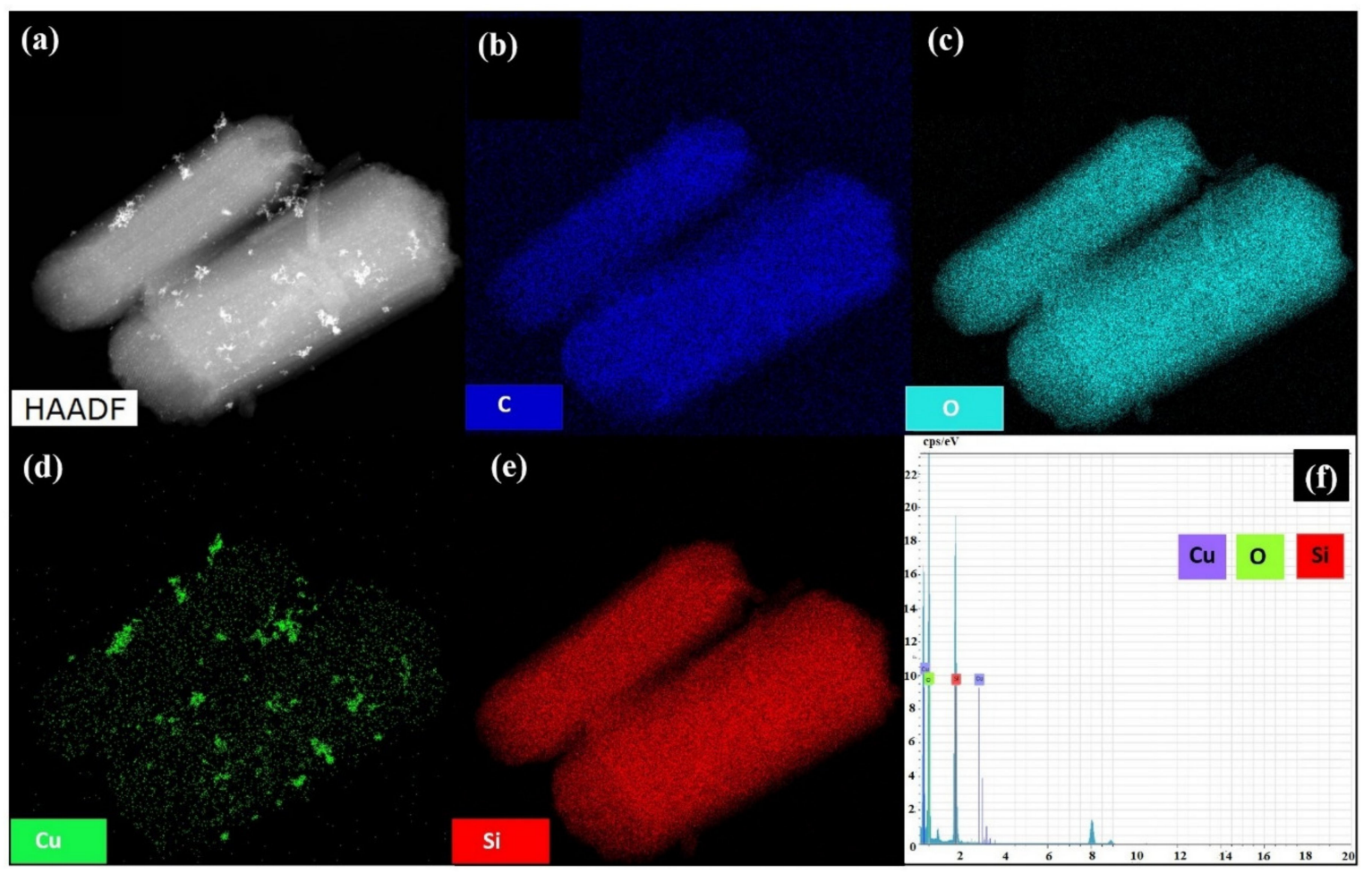

Figure 5. HAADF-TEM (a) Energy-dispersive X-ray spectroscopy (EDX) mapping images; (b-e) and spectrum; (f) of CuNPs/p(DMAEMA-co-TBA)/TSBA.

The small angle X-ray scattering (SAXS) patterns of materials are displayed in Figure 6. The SAXS patterns of pristine SBA-15 show three well-resolved peaks at a scattering vector, i.e., $\mathrm{q}^{*}=0.07,0.12$, and $0.14 \AA^{-1}$, indexed to the (100), (110), and (220) reflection planes, respectively, which are associated with the 2D hexagonal pore arrangement of SBA-15. The structure of SBA-15 remained after the surface modification with TMSPM for TSBA, although a slight decrease in the intensity for the d spacing (110), (200), and (100) peaks was observed. The introduction of the copolymer to DMAEMA and TBA could slightly affect the mesoporous structure of SBA-15. After the grafting of the copolymer (i.e., p(DMAEMA-co-TBA)/TSBA), the intensities of the (110) and (200) peaks decreased, and the peak showing a maximum shifted slightly toward the right side, which demonstrates the diminishing of the ordered mesoporous structure due to the covering of the copolymer on the internal and external surface of TSBA, followed by a decrease in the pore size of the materials. 


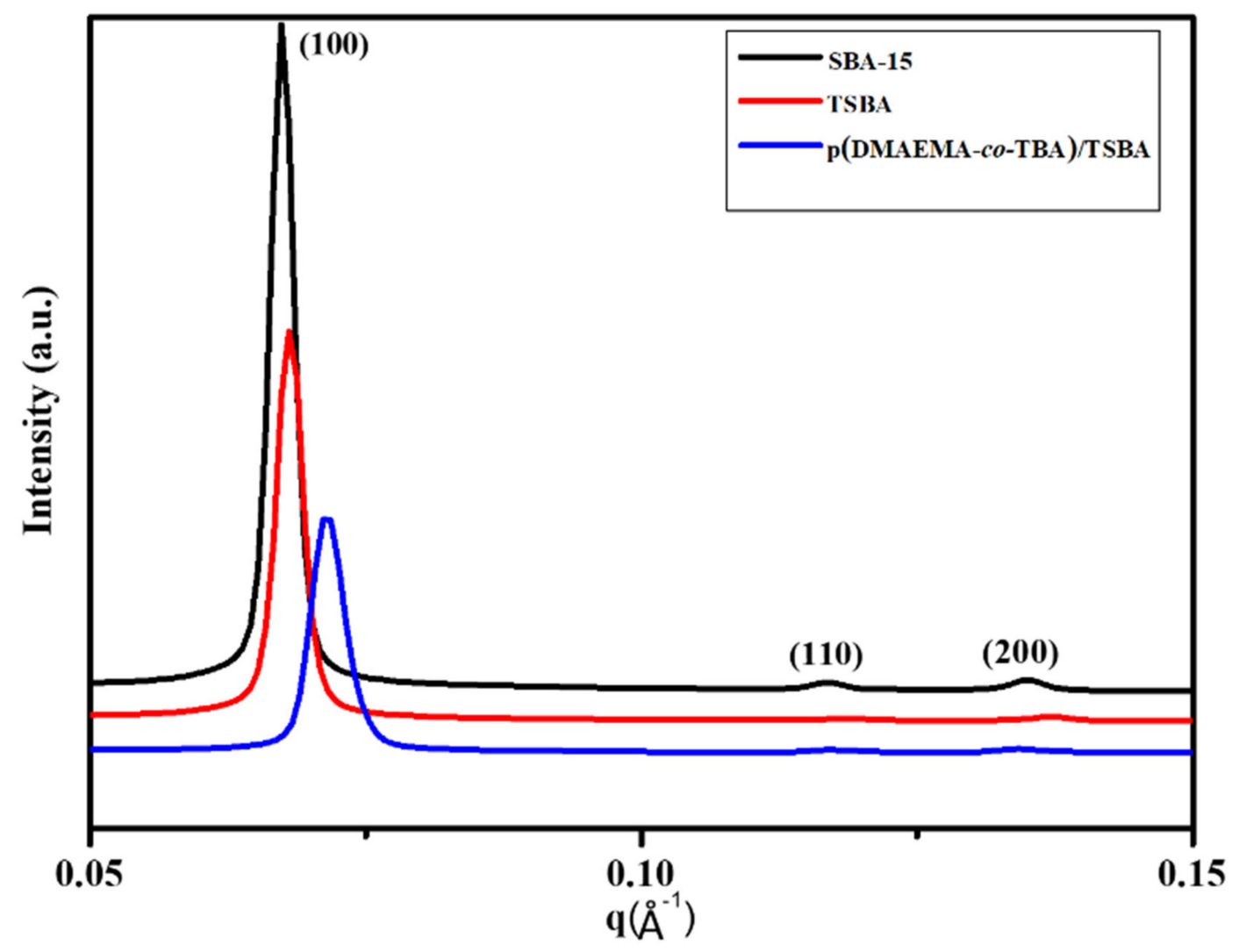

Figure 6. SAXS patterns of SBA-15, TSBA, and $\mathrm{p}(\mathrm{DMAEMA-co-TBA}) / \mathrm{TSBA}$.

Wide angle XRD (WAXRD) patterns were recorded with the aim of confirming the presence of the crystalline CuNPs in the CuNPs/p(DMAEMA-co-TBA)/TSBA catalyst. Figure S1 illustrates the diffraction patterns of the catalyst. The catalyst exhibited typical diffraction patterns of CuNPs, with a small broad peak at approximately $2 \theta=43^{\circ}, 50^{\circ}$, and $73^{\circ}$, which corresponded to the (111), (200), and (220) crystal planes of CuNPs, respectively. The WAXRD pattern presents intense diffraction peaks in the range $2 \theta=30-80^{\circ}$, representing monoclinic $\mathrm{CuO}$. A broad diffraction peak of cuprite (111) was also observed at a diffraction angle of $36.2^{\circ}$ (Figure S1). These diffraction peaks were similar in terms of angular positions to that of $f c c$ pure bulk copper crystalline peaks, but were relatively broad $[29,30]$. We have calculated the particle size of CuNPs present in the CuNPs/p(DMAEMA-co-TBA)/TSBA catalyst and CuNPs/TSBA. The average particle size of the nanoparticles is around $1.8 \mathrm{~nm}$ for CuNPs/p(DMAEMA-co-TBA)/TSBA catalyst and CuNPs/TSBA, which is determined from the HRTEM image using the Image J software as shown in Figures S2 and S3 as well as from the Scherer's equation [31]. The mean size of CuNPs estimated from Scherer's equation was in good agreement with the data taken from the HRTEM image. In the case of CuNPs/SBA-15, however, the phases $\mathrm{Cu}_{2} \mathrm{O}$ and $\mathrm{CuO}$ were undetected. Peaks attributed to copper nanoparticles were not observed in the WAXRD pattern for CuNPs/SBA-15, which can be explained by the high dispersion of the copper oxide nanoparticles on the surface of SBA-15, which makes them undetectable by XRD pattern [30].

Figure $\mathrm{S} 4$ indicates the nitrogen adsorption-desorption isotherms for SBA-15, TSBA, and poly (DMAEMA-co-TBA)/TSBA, which exhibit typical type IV isotherms with $\mathrm{H} 1$ hysteresis loops as defined by IUPAC for all three materials, indicating that all three materials possess a mesoporous structure. The physico-chemical parameters of the samples are summarized in Table S1. The prepared SBA- 15 had a Brunauer-Emmett-Teller (BET) surface area of $708 \mathrm{~m}^{2} \mathrm{~g}^{-1}$, pore volume of $0.89 \mathrm{~cm}^{3} \mathrm{~g}^{-1}$, and pore size of $7.7 \mathrm{~nm}$. For functionalized SBA-15 (TSBA), the BET surface area, pore volume, and pore size were reduced to $600 \mathrm{~m}^{2} \mathrm{~g}^{-1}, 0.65 \mathrm{~cm}^{3} \mathrm{~g}^{-1}$, and $6.8 \mathrm{~nm}$, respectively. The variables agree with those reported in previous literature [27]. A higher BET surface area for the channels was observed in the SBA-15 
samples. The existence of functional groups at the mesoporous surface promotes copolymerization on the surface. The results show that pore volume, pore size, and surface area are decreased after functionalization. This confirms that the functional group is located not only on the outer surface but also inside the mesoporous channel. After polymerization (i.e., p(DMAEMA-co-TBA)/TSBA), the surface area, pore volume, and pore size further decreased to $353 \mathrm{~m}^{2} \mathrm{~g}^{-1}, 0.46 \mathrm{~cm}^{3} \mathrm{~g}^{-1}$, and $6.1 \mathrm{~nm}$, respectively. The noticeable decrease in the physico-chemical parameters of the polymer-coated samples evidences the successful grafting of DMAEMA and TBA on the surface of SBA-15 [26,32].

In the thermogravimetric analysis (TGA) curves of Figure 7, the SBA-15, TSBA, and p(DMAEMAco-TBA)/TSBA samples were heated from $25^{\circ} \mathrm{C}$ to $800{ }^{\circ} \mathrm{C}$ at a rate of $10^{\circ} \mathrm{C} / \mathrm{min}$ under a $\mathrm{N}_{2}$ atmosphere. The main weight loss in the first region (below ca. $100{ }^{\circ} \mathrm{C}$ ) originates from desorption of the physically adsorbed water on the surface. The mass loss was 3\%, which can be considered a negligible value due to the very small rate of mass loss at the temperature. The calcined SBA-15 indicates complete removal of the surfactant during the calcination process, which proves the thermal stability of SBA-15. The weight loss up to $800{ }^{\circ} \mathrm{C}$ was found to be $15 \%$ after the surface modification of SBA-15 with TMSPM [26], whereas the weight loss at approximately $350-450{ }^{\circ} \mathrm{C}$ may be due to the degradation of TMSPM. Similarly, an additional $10 \%$ loss of the sample up to $800{ }^{\circ} \mathrm{C}$, along with the weight loss at $200-350{ }^{\circ} \mathrm{C}$, may be due to the decomposition of the copolymer, which indicates a reasonable amount of copolymer loading on the SBA-15 surface.

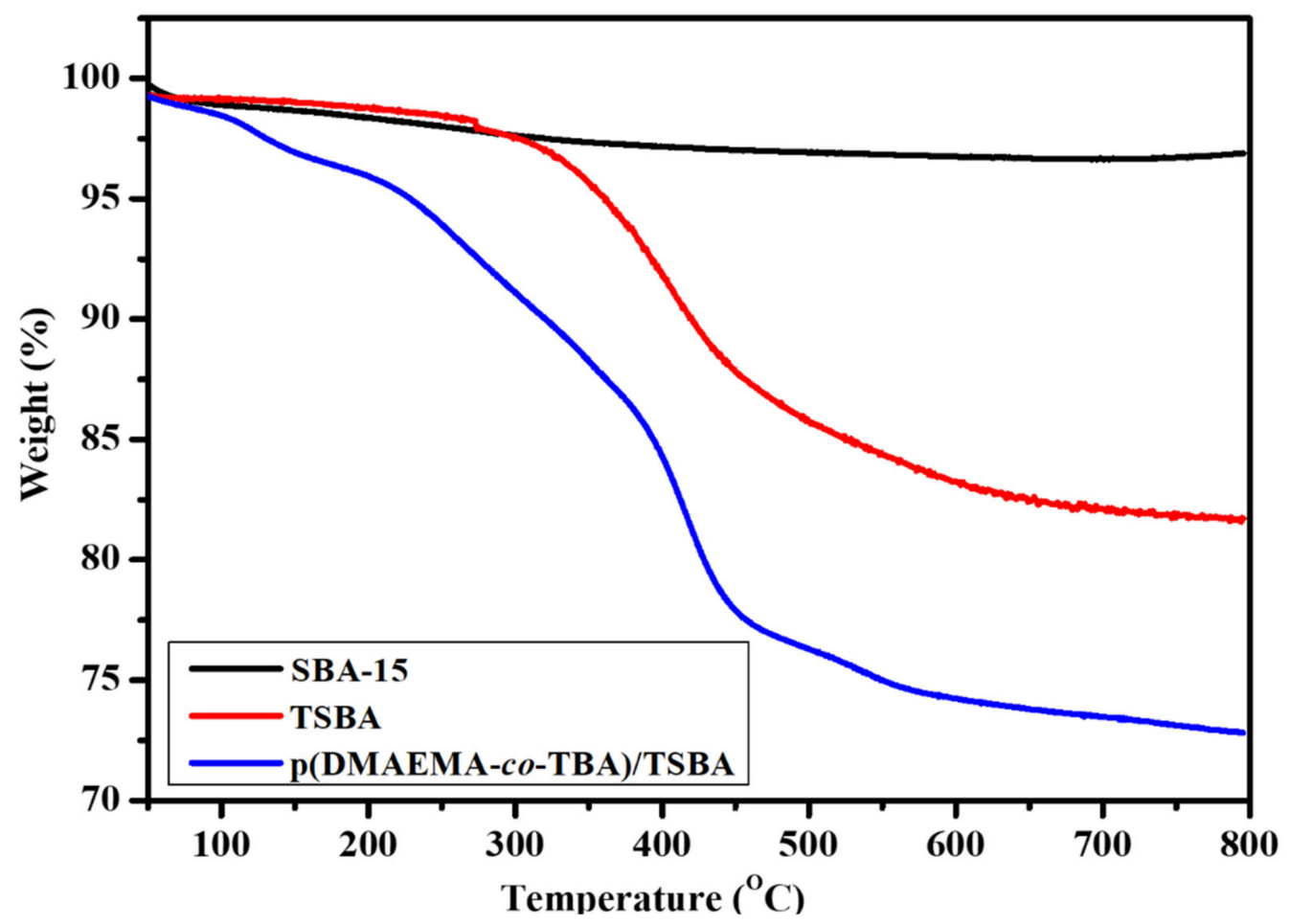

Figure 7. Thermogravimetric analysis (TGA) curves of SBA-15, TSBA, and p(DMAEMA-co-TBA)/TSBA.

\subsection{Stimuli-Responsive Performance}

The temperature and $\mathrm{pH}$ dependent changes in solution behavior of the dual responsive copolymer grafted catalyst CuNPs/p(DMAEMA-co-TBA)/TSBA were studied using dynamic light scattering (DLS). We evaluated how the thermo-responsive monomer TBA influences the transition temperature or LCST of the copolymers, or how the presence of the hydrophobic co-monomer influences the self-assembly of the DMAEMA chains on mesoporous silica. To explore the temperature-responsive properties of the catalyst, the hydrodynamic diameters $\left(\mathrm{D}_{\mathrm{H}}\right)$ of the $\mathrm{CuNPs} / \mathrm{p}$ (DMAEMA-co-TBA)/TSBA in aqueous solution at $\mathrm{pH} 7$ at different temperatures were measured. Unless otherwise specified, p(DMAEMA-co-TBA)(6:4) was used 
throughout the text, where 6:4 stands for the comonomer ratio of DMAEMA to TBA, as described in the Section 2.1. The hydrodynamic diameter of CuNPs/p(DMAEMA-co-TBA)/TSB with different comonomer ratios was plotted as a function of temperature at $\mathrm{pH} 7$ in Figure S5. In Figure 8a, the hydrodynamic diameter of $\mathrm{CuNPs} / \mathrm{p}$ (DMAEMA)/TSBA as a function of temperature is given as a reference, where the comonomer ratio of DMAEA to TBA is 100:0.

The LCST of the CuNPs/p(DMAEMA-co-TBA)/TSBA decreased with increasing TBA content in the copolymer, as shown in Figure S5. For instance, the LCST of the CuNPs/p(DMAEMA-co-TBA)(6:4)/TSBA in aqueous solution was found to be in the range of $25-30{ }^{\circ} \mathrm{C}$, whereas the $\mathrm{CuNPs} / \mathrm{p}$ (DMAEMA)/TSBA particles displayed a fine transition in the range of $45-50^{\circ} \mathrm{C}[27,33]$ (Figure 8 and Figure S5). Upon increasing the temperature from 20 to $50{ }^{\circ} \mathrm{C}$, the hydrodynamic diameter of CuNPs/p(DMAEMA-co-TBA)(6:4)/TSBA particles noticeably decreased from 962 to $761 \mathrm{~nm}$, which can be attributed to the hydrophobic interactions and hydrogen bonding. At low temperatures, the fragments arranged into a random coil conformation due to intermolecular hydrogen bonding interactions. As the temperature increased, the copolymer segments were dehydrated, and the intermolecular hydrophobic interactions dominated, resulting in the collapse of the copolymer segments on the surface of SBA-15. The LCST of p(DMAEMA) was lowered by copolymerization with a hydrophobic co-monomer (TBA). The LCST of the copolymer was found to be in the range of $25-30{ }^{\circ} \mathrm{C}$. These results demonstrate the copolymerization of the TBA and DMAEMA monomers, which broadened and lowered the LCST $[33,34]$. This can be attributed to the fact that TBA content changes the molecule's hydrophobicity. Obviously, the presence of the comonomer decreases the LCST, as previously reported.

(a)

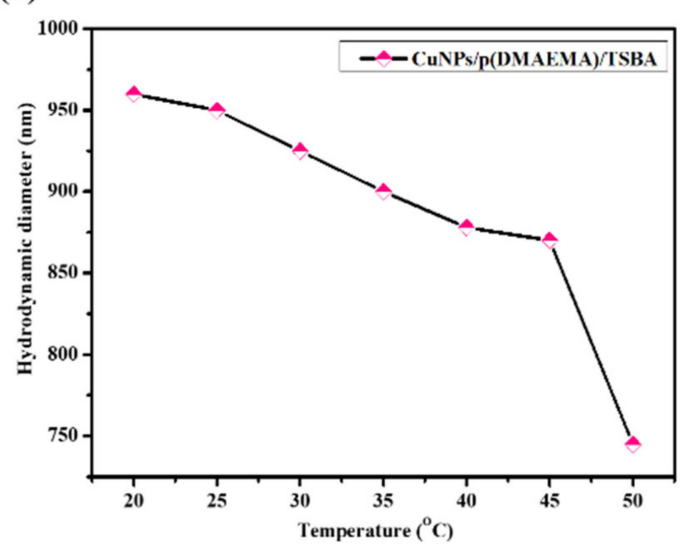

(b)

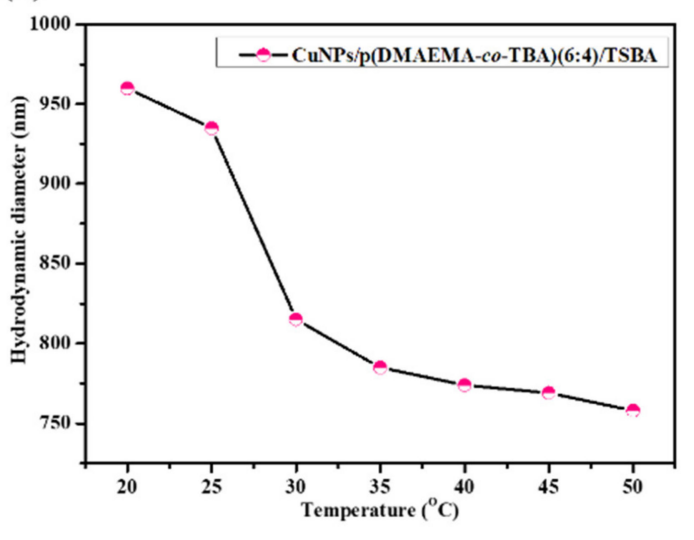

Figure 8. Particle size of (a) CuNPs/p(DMAEMA)/TSBA and (b) CuNPs/p(DMAEMA-co-TBA)(6:4) /TSBA as a function of temperature at $\mathrm{pH}=7$.

The $\mathrm{pH}$-responsive behavior of CuNPs/p(DMAEMA-co-TBA)/TSBA and CuNPs/p(DMAEMA)/TSBA was also investigated. The hydrodynamic diameter $\left(\mathrm{D}_{\mathrm{H}}\right)$ was measured at different $\mathrm{pH}$ values ranging from 2 to 10 , as shown in Figure 9. At $\mathrm{pH}<8$, the polymer chains are protonated and reach a maximum swelling of $1110 \mathrm{~nm}$. The hydration layer rises on the outer surfaces of SBA-15, which results in a larger particle diameter measurement. The changes in the hydrodynamic diameter of CuNPs/p(DMAEMA-co-TBA)/TSBA and CuNPs/p(DMAEMA)/TSBA at different $\mathrm{pH}$ values showed a pKa between 7 and 8 , which agrees with previous literature [26]. Under acidic conditions, the polymer was stretched and in an opened state due to the protonation of tertiary amine functional groups in p(DMAEMA), owing to the extension of polymer chains. The repulsion between the positive charges formed on the amino groups $\left(\mathrm{NH}_{3}{ }^{+}\right)$was recognized on the copolymer with DMAEMA, which is responsible for the larger particle sizes observed at $\mathrm{pH}$ values of 2-6. Therefore, the reactants were able to diffuse into the pores of the nanocarriers. When the $\mathrm{pH}$ value increases, the amino groups are deprotonated $\left(\mathrm{NH}_{2}\right)$, which results in an increase in the hydrophobic interaction; therefore, the $\mathrm{D}_{\mathrm{H}}$ of the catalyst decreases due to deprotonation of the side ammonium group 
of the polymer. At $\mathrm{pH} \mathrm{10,} \mathrm{the} \mathrm{compact} \mathrm{polymer} \mathrm{layer} \mathrm{blocks} \mathrm{the} \mathrm{pores.} \mathrm{This} \mathrm{behavior} \mathrm{was} \mathrm{attributed} \mathrm{to}$ the increased hydrophilicity resulting from the ionization of the DMAEMA component taking place along the TBA chains [34].

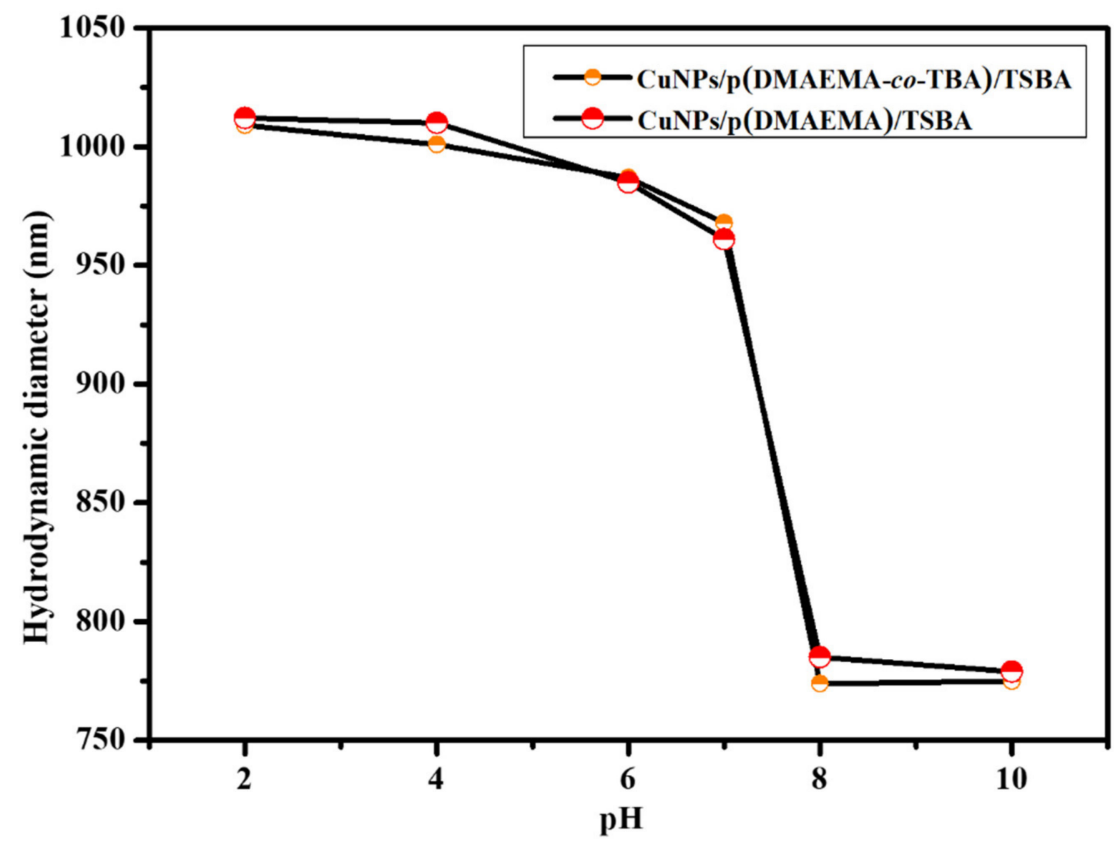

Figure 9. Hydrodynamic diameter of CuNPs/p(DMAEMA-co-TBA)/TSBA and CuNPs/p(DMAEMA)/TSBA at different $\mathrm{pH}$ values, as measured by dynamic light scattering (DLS).

\subsection{Catalysis}

\subsubsection{Optimization}

To explore the catalytic behavior, the efficient reusable mesoporous silica-supported CuNPs/p(DMAEMA-co-TBA)/TSBA catalyst was activated by heating at $50{ }^{\circ} \mathrm{C}$ overnight and used for the oxidation of benzyl alcohol under mild conditions. The catalytic system was optimized using different variables: oxidants, temperature, solvent, and catalytic amounts. Initially, to evaluate the reaction conditions for the selective oxidation of alcohols, we used benzyl alcohol as a model substrate.

Good conversions were achieved for dimethyl sulfoxide (DMSO), toluene, and water, but $\mathrm{H}_{2} \mathrm{O}$ was more eco-friendly than DMSO and toluene (Table S2). Among the oxidants, we found that $\mathrm{H}_{2} \mathrm{O}_{2}$ was more promising than other oxidizing agents, such as $\mathrm{H}_{5} \mathrm{IO}_{6}$ and $t$ - $\mathrm{BuOOH}$. In addition, it was found that $1.0 \mathrm{mmol}$ of $\mathrm{H}_{2} \mathrm{O}_{2}$ was sufficient for maximum conversion (Table S3). Therefore, the activity of the CuNPs/p(DMAEMA-co-TBA)/TSBA catalyst for the oxidation of benzyl alcohols was investigated using $\mathrm{H}_{2} \mathrm{O}_{2}$ as oxidant throughout this work. In our future work, however, we will use molecular oxygen as oxidant because $\mathrm{O}_{2}$ is cheaper and an environmentally friendly oxidant than $\mathrm{H}_{2} \mathrm{O}_{2}$ to further reveal the catalytic activity of the present catalyst.

It is also noteworthy that the conversion of benzaldehyde increased steadily with an increase of the catalyst amount. Furthermore, the catalytic conversion reached a maximum when 5 mg of catalyst was used. Increasing the amount of catalyst above $5 \mathrm{mg}$ did not show any noticeable influence on the reactivity (Table $\mathrm{S} 4$ ).

The blank reaction was performed under identical conditions. The yield of benzaldehyde was only $8 \%$ in the control experiment without any catalyst or oxidant (Table S5, entry 12). In the absence of the oxidant, CuNPs/p(DMAEMA-co-TBA)/TSBA catalysts alone showed only a $41 \%$ yield of benzaldehyde (Table S3, entry 7), and in the absence of a catalyst, 18\% (Table S5, entry 1) benzaldehyde was produced, indicating that $\mathrm{H}_{2} \mathrm{O}_{2}$ alone cannot oxidize benzyl alcohol into benzaldehyde. To increase the benzaldehyde 
yield, such promoters as oxidants and catalysts were added. When the reaction was carried out with pristine SBA-15 only (without CuNPs and copolymer), p(DMAEMA-co-TBA)/TSBA (without CuNPs), or $\mathrm{CuNPs} / \mathrm{p}$ (DMAEMA-co-TBA) (without TSBA), 21\%, 35\%, 65\%, conversion, respectively, of benzyl alcohol was achieved. This result hints that both the polymer and copper species must be efficient active sites for the reaction and that the presence of a suitable support is essential for a good catalysis (Table S5, entries 2, 8, 11). We conducted experiments using CuNPs/SBA-15 without a functionalized polymer, which resulted in moderate conversion (Table S5, entry 3). It should be noted here that if the actual loading of $\mathrm{Cu}$ for CuNPs/SBA-15 is lower than that of CuNPs/pDMAEMA-co-TBA)/TSBA, it cannot be said that the latter catalyst has higher catalytic activity than former one. As mentioned from the WAXRD pattern in Figure S1, however, the actual loadings of CuNPs of CuNPs/SBA-15 were hard to be estimated. Thus, even if direct comparison of the catalytic activities of these two catalysts may have some ambiguity, it is of no doubt the fact that the CuNPs/p(DMAEMA-co-TBA)/TSBA catalyst showed the highest activity with $99 \%$ of yield, whereas the CuNPs/SBA-15 did not improve the conversion $(80 \%)$, which may be attributed to the synergetic effect of both polymer and CuNPs. The yield was significantly lower in the absence of CuNPs in the catalyst p(DMAEMA)/TSBA (Table S5, entry 4). To study the effect of the copolymer coating, the experiment was conducted with and without the TBA monomer at different temperatures $\left(25\right.$ and $50^{\circ} \mathrm{C}$ ) in the presence of CuNPs (Table S5, entries 5 and 6). Interestingly, the results indicated the importance of TBA, with a slight increase in the conversion after copolymerization at room temperature. Even in the presence of CuNPs, $\mathrm{p}$ (DMAEMA) without TBA monomer showed low conversion (60\%) (Table S5, entry 7). The influence of other reaction conditions on the catalytic performance was investigated.

The temperature-responsive environment of the catalyst was predicted from the DLS measurements. Then, we carried out the reaction under different temperature conditions, i.e., above and below the LCST. The LCST of CuNPs/p(DMAEMA)/TSBA was $45-50^{\circ} \mathrm{C}$. After copolymerization between DMAEMA and TBA on SBA-15, the LCST value decreased to $25-30{ }^{\circ} \mathrm{C}$. Therefore, we tested the oxidation reaction at room temperature (below LCST) and $50^{\circ} \mathrm{C}$ (above LCST), and found that benzyl alcohol was converted to benzaldehyde at rates of $99 \%$ and $91 \%$, respectively (Table S5, entries 9 and 10). This provides clear evidence that the shrinking property was much higher for the catalyst at $50{ }^{\circ} \mathrm{C}$. On shrinking, the polymer-coated $\mathrm{CuNPs/p}$ (DMAEMA-co-TBA)/TSBA behaves like a wall and obstructs the outgoing of reactants to the metal surface inside the pores, which negatively affects the reaction rate. Below the LCST, the polymer enlarges and opens the pores of mesoporous silica SBA-15, which leads to better interaction between the active sites of the CuNPs inside the pores and the reactants facing, resulting in better organic conversion. DLS studies showed that the catalyst swelled at acidic $\mathrm{pH}$. The oxidation reaction of benzyl alcohol was investigated under different $\mathrm{pH}$ conditions to study the $\mathrm{pH}$-responsive behavior of the catalyst. The repulsion between the positive charges in $\mathrm{NH}_{3}{ }^{+}$was large, and the swelling property was enhanced at low $\mathrm{pH}$, which resulted in better conversion (99\%) (Table S5, entry 10).

\subsubsection{Extension of Scope}

The scope of the CuNPs/p(DMAEMA-co-TBA)/TSBA-catalyzed oxidation of various substituted alcohols was examined (Table 1). The results indicate that aromatic alcohols usually require less reaction time and result in good conversion compared to aliphatic alcohols due to the good interaction between the aromatic alcohols and catalyst surface through $\pi-\pi$ bonding [35,36]. The active oxidation of benzyl alcohol is due to the active phenyl group. Benzaldehyde was the main oxidation product in this system with $99 \%$ conversion and $99 \%$ selectivity (Table 1, entry 1). Various mono-substituted benzyl alcohols were utilized to produce aldehydes and ketones in high yields (Table 1, entries 1-15). In certain catalytic systems, it was not easy to obtain the desired benzaldehyde in the oxidation of benzyl alcohol, but benzoic acid was often produced instead of benzaldehyde. Ronzer et al., reported $\mathrm{Na}_{2}\left[\mathrm{WZnZn} \mathrm{Zn}_{2}\left(\mathrm{H}_{2} \mathrm{O}\right)_{2}\left(\mathrm{ZnW}_{9} \mathrm{O}_{34}\right)_{2}\right.$ catalyst that oxidized primary alcohols to the benzyl alcohol as well as other corresponding carboxylic acids [37]. 
Table 1. Oxidation of alcohols using copper nanoparticles (CuNPs)/p(DMAEMA-co-TBA)/TSBA catalyst ${ }^{\text {a }}$.

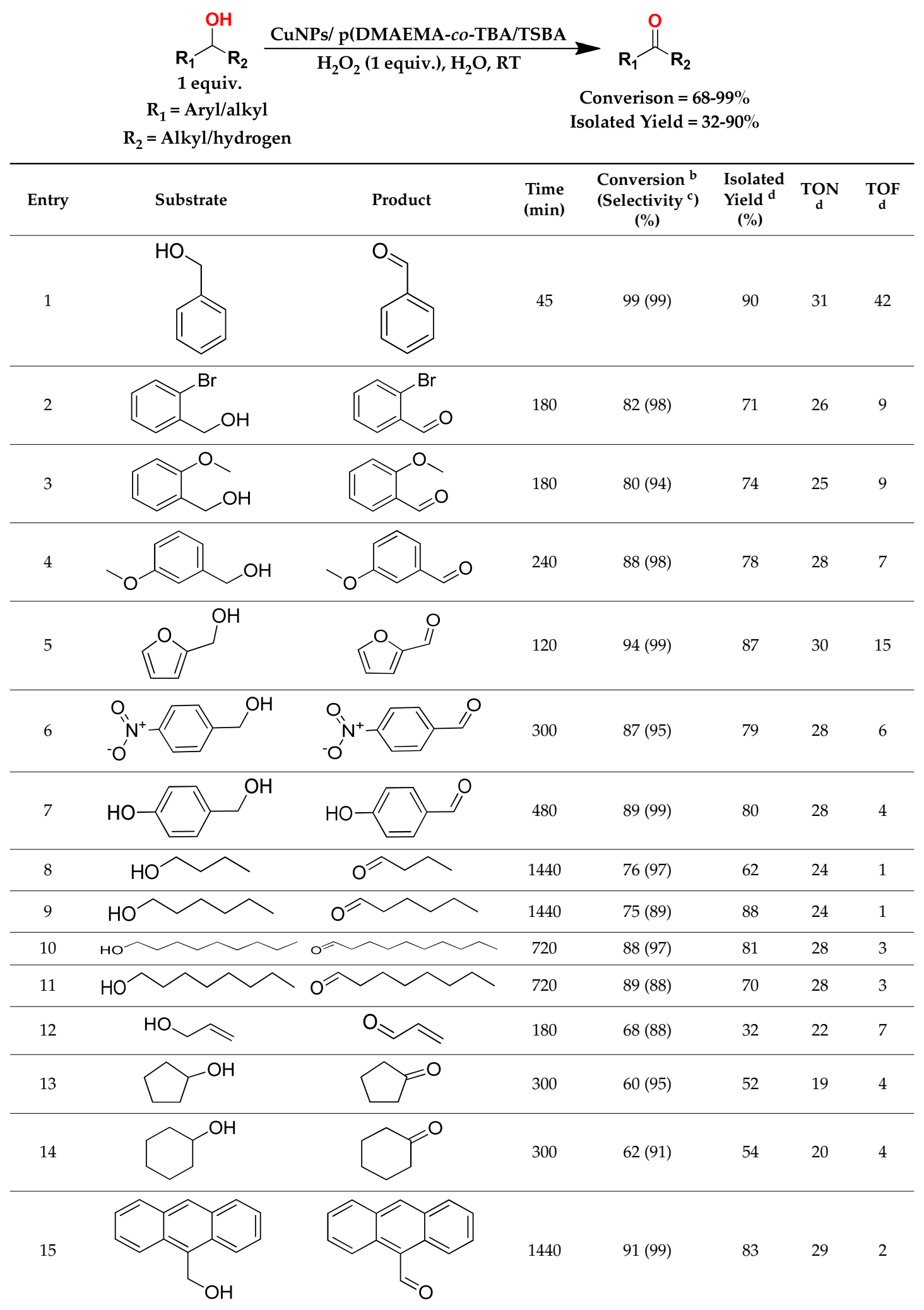

${ }^{a}$ Reaction conditions: Substrate $(1 \mathrm{mmol})$, catalyst $(5.0 \mathrm{mg}), \mathrm{H}_{2} \mathrm{O}_{2}(1 \mathrm{mmol})$, water $(5 \mathrm{~mL}), 25^{\circ} \mathrm{C} .{ }^{\mathrm{b}}$ Determined by GC. ${ }^{c}$ Selectivity in the parentheses were calculated from GC. ${ }^{d}$ Turnover number (TON) and turnover frequency (TOF) were calculated according to the reference [38]. 
Ortho-substituted benzyl alcohols produced the desired aldehydes (Table 1, entries 2 and 3) in lower yields, in part due to steric hindrance. Additionally, a heterocyclic system could achieve better conversion than aromatic substituted alcohols with good selectivity (Table 1, entry 5). The decrease in the yield was caused by the substitution of strong electron-withdrawing nitro and $\mathrm{OH}$ substituents (Table 1, entries 6 and 7), whereas halogen substituents produced a modest yield (Table 1, entry 2).

Unfortunately the non-benzylic primary alcohols such as 1-butanol and 1-hexanol gave less conversion than that of the non-benzylic alcohols with more alkyl chains (Table 1, entries 8 and 9). The rate of conversion increased with the increasing chain length (Table 1, entries 10 and 11). When the catalyst was used in an allyl alcohol oxidation, the desired $\alpha, \beta$-unsaturated aldehyde was produced with a $32 \%$ yield (Table 1, entry 12). To expand the substrate scope, the oxidation of cyclic alcohols, such as cyclohexanol and cyclopentanol, was also tested, which are also quantitatively converted to their corresponding aldehydes and ketones with very good yields (Table 1, entries 13 and 14). All the corresponding aldehydes were produced with high selectivity, and over-oxidation into carboxylic acid was not observed [39]. ${ }^{1} \mathrm{H} /{ }^{13} \mathrm{C}$ NMR spectra of oxidized products are included in the Supplementary Data (Figure S6). Turnover number (TON) and turnover frequency (TOF) values are also included in Table 1. TOF ranged from 1 to 42 for the oxidation of alcohols under aqueous conditions.

To demonstrate the advantages of CuNPs/p(DMAEMA-co-TBA)/TSBA as a heterogeneous catalyst in this reaction, our results were compared with other homogeneous and heterogeneous catalysis of this reaction in the literature [27] (Table 2). TON and TOF values are also included in Table 2 for better comparison of catalytic activity of various catalysts. TOF ranged from 2 to 460 for the oxidation of benzyl alcohols under various reaction conditions. The TON and TOF values of the $\mathrm{CuNPs/p}$ (DMAEMA-co-TBA)/TSBA catalyst were found to be comparable with previous reported literatures. The results show that our catalyst is superior to or at least comparable with other catalysts in terms of the yields and reaction times.

\subsection{Reusability of the Catalyst}

One of the major reasons for supporting metal nanoparticles incorporated onto polymer-coated mesoporous SBA-15 is to enhance the activity of the catalyst. In addition to reaction conditions such as reaction temperature and time, recyclability is also crucial for evaluating catalysts. For this, recycling experiments were performed on the CuNPs/p(DMAEMA-co-TBA)/TSBA catalyst. To study the stability and reusability of the heterogeneous catalysts, they were recovered through centrifugation, washed with ethanol, dried at $50^{\circ} \mathrm{C}$ for $5 \mathrm{~h}$, and used in the recycling study. The yield and structure of the final products were compared with those tested for the fresh catalyst. The obtained results of the catalysts indicate that the catalytic activity decreased after recycling (Figure 10). It may be assumed that the slight deactivation of the catalyst after recycling might be due to the loss of some catalyst during recycling test as well as the oxidation of fouling or aging of the catalyst. For instance, hydroxyl radicals may damage the polymeric structure on the surface of the hybrid materials after the fifth wash, which may potentially impact the catalytic performance. In addition, metal leaching may take place during continuous washing and recycling for further use.

WAXRD patterns indicate that there were no changes in the crystallinity of the CuNPs after the oxidation reaction, as shown in Figure S7. FETEM analysis clarifies the presence of CuNPs both inside and outside the pores. The black spots indicate the presence of CuNPs (Figure S8). FT-IR analysis was also conducted after the fifth cycle of the catalyst to investigate the presence of functional groups (Figure S9). Two weak peaks observed at 2916 and $2848 \mathrm{~cm}^{-1}$ prove the existence of C-H stretching, which was associated with the aliphatic group. A strong peak at $1728 \mathrm{~cm}^{-1}$, corresponding to the $\mathrm{C}=\mathrm{O}$ group confirms that the catalyst structure was retained after the synthesis of aldehyde during the reaction. It is interesting to note that the CuNPs/p(DMAEMA-co-TBA)/TSBA catalyst for this work was found to be more cost-effective for preparation than simple metal nanoparticles such as CuNPs and FeNPs by our rough estimation, as shown in Tables S6-S9. 
Table 2. Comparison of catalytic activities of the oxidation of benzyl alcohol for catalysts reported in literature and this work.

\begin{tabular}{|c|c|c|c|c|c|c|c|c|c|}
\hline Entry & Catalyst & Oxidant & $\begin{array}{c}\text { Catalyst } \\
\text { Amount } \\
\text { (mg) }\end{array}$ & $\begin{array}{l}\text { Time } \\
\text { (h) }\end{array}$ & $\begin{array}{l}\text { Temp. } \\
\left({ }^{\circ} \mathrm{C}\right)\end{array}$ & $\begin{array}{l}\text { Yield } \\
(\%)\end{array}$ & TON & TOF & Ref. \\
\hline 1 & $\mathrm{Cu}\left(\mathrm{NO}_{3}\right)_{2}$ & $\mathrm{H}_{2} \mathrm{O}_{2}(1.2 \mathrm{~mL})$ & 10 & 1.5 & 80 & 52 & 78 & 52 & [40] \\
\hline 2 & Pd@Cu(II)-MOF & $\mathrm{O}_{2}$ & 23 & 25 & 130 & 99 & 50 & 2 & [41] \\
\hline 3 & $\mathrm{CuSO}_{4}$ & $\mathrm{H}_{2} \mathrm{O}_{2}(1 \mathrm{~mL})$ & 10 & 1.5 & 80 & 62 & 186 & 124 & [42] \\
\hline 4 & $\mathrm{CuCl}_{2} / \mathrm{CH}_{3} \mathrm{COCH}_{3}$ & $\mathrm{O}_{2}$ & 25 & 1 & 25 & 95 & 190 & 190 & [43] \\
\hline 5 & $\mathrm{CuMn}_{2}$ oxide & $\mathrm{O}_{2}$ & 200 & 0.6 & 102 & 99 & - & - & [44] \\
\hline 6 & $\mathrm{CuCl}_{2} / \mathrm{THF}$ & $\mathrm{O}_{2}$ & 20 & 2 & 80 & 85 & 43 & 22 & [45] \\
\hline 7 & $\mathrm{CuSH} / 1 \mathrm{NaK}$ & $t-\mathrm{BuOOH}(1.6 \mathrm{~mL})$ & 20 & 24 & 70 & 72 & 165 & 7 & [46] \\
\hline 8 & $\mathrm{Au}-\mathrm{Cu} / \mathrm{SiO}_{2}$ & $\mathrm{O}_{2}$ & 200 & 10 & 319 & 92 & 702 & 280 & [47] \\
\hline 9 & $\mathrm{AgCu} / \mathrm{SiC}$ & $\mathrm{O}_{2}$ & 280 & 2.5 & 280 & 99 & 1151 & 460 & [48] \\
\hline 10 & $\begin{array}{c}\text { CuNPs/p(DMAEMA- } \\
\text { co-TBA)/TSBA }\end{array}$ & $\mathrm{H}_{2} \mathrm{O}_{2}(1 \mathrm{~mL})$ & 5.0 & 1 & 25 & 99 & 31 & 42 & $\begin{array}{l}\text { This } \\
\text { work }\end{array}$ \\
\hline
\end{tabular}

Note: $\mathrm{Cu}\left(\mathrm{NO}_{3}\right)_{2}$ : copper nitrate catalyst with the presence of $\mathrm{H}_{2} \mathrm{O}_{2}$ oxidant, without adding any additive. Pd@Cu (II)-MOF: The metal-organic zeolite imidazolate framework-8 supported palladium-copper bimetallic catalysts with a core-shell structure. $\mathrm{CuSO}_{4}$ : copper sulphate catalyst with the presence of $\mathrm{H}_{2} \mathrm{O}_{2}$ oxidant, without adding any additive. $\mathrm{CuCl}_{2} / \mathrm{CH}_{3} \mathrm{COCH}_{3}$ : Photo catalytic oxidation of benzyl alcohol by homogeneous $\mathrm{CuCl}_{2}, \mathrm{CuMn}_{2}$ oxide: copper-manganese mixed oxide nanoparticles prepared by co-precipitation method for selective oxidation of benzyl alcohol using molecular oxygen as an oxidant, $\mathrm{CuCl}_{2} / \mathrm{THF}$ : The catalytic system consists of $\mathrm{CuCl}_{2}$ and 2,2'-biquiniline 4-4' dicarboxylic acid dipotassium salt (BQC) and was used for selective oxidation, $\mathrm{CuSH} / 1 \mathrm{NaK}$ : oxidation in the presence of the crystalline copper silicates $\mathrm{CuSH}-1 \mathrm{NaK}, t-\mathrm{BuOOH}$ oxidant and acetonitrile solvent, $\mathrm{Au}-\mathrm{Cu} / \mathrm{SiO}_{2}$ : This bimetallic gold-copper on silica catalyst was prepared in a simple impregnation, $\mathrm{AgCu} / \mathrm{SiC}$ : The synergistic effect between silver and oxide of $\mathrm{Cu}_{2} \mathrm{O}$ is explained and is supported with silicon carbide, CuNPs/p(DMAEMA-co-TBA)/TSBA: Copper nanoparticle decorated on copolymerized SBA-15 mesoporous silica material.

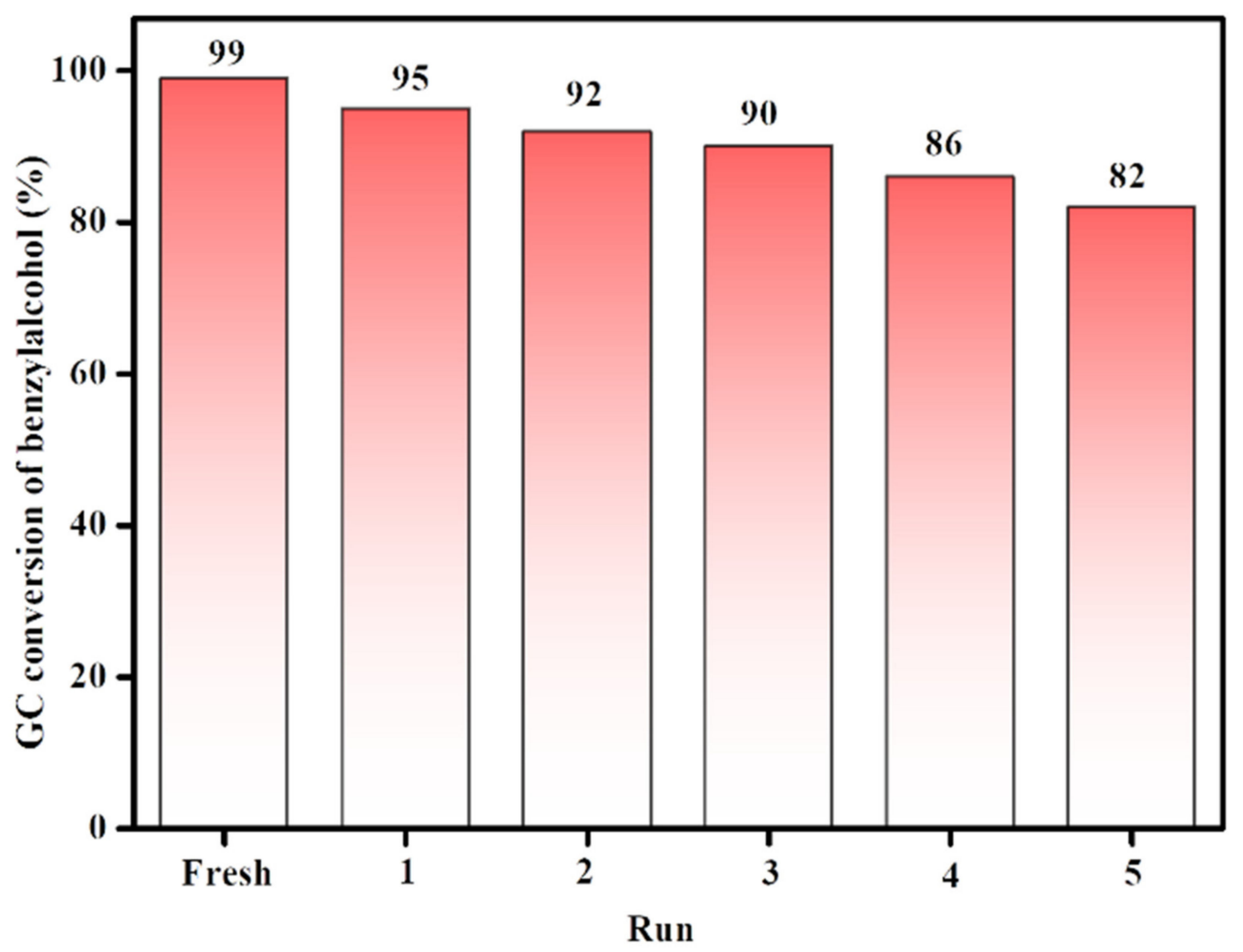

Figure 10. Recyclability of CuNPs/p(DMAEMA-co-TBA)/TSBA hybrid catalyst on the oxidation of alcohols. 
Before concluding, it should be noted that studies on the degradation mechanism and kinetics are quite needed to understand the catalytic activity for oxidation of various alcohols in depth. According to previous reports, for instance, the reaction of $\mathrm{H}_{2} \mathrm{O}_{2}$ with $\mathrm{CuNPs}$ under acidic or neutral conditions generates more hydroxyl radicles, which will increase the rate of the oxidation reaction [49,50]. In this work, the $\mathrm{pH}$ of the reacting mixture was about 7 , which may enhance the catalytic activity. Thus further works on the subject should be conducted in order to interpret the results in this work in more depth for our future work. In this sense, our future work will include the degradation kinetics, i.e., the removal rate as a function of time, which is a key information to compare with other catalysts, as well as the degradation mechanism by the verification of radical species with the typical scavenger results, so that we could reveal whether hydroxyl radical is the main oxidant for alcohols degradation or not, etc.

\section{Conclusions}

In this study, CuNPs were successfully immobilized on a temperature- and $\mathrm{pH}$-responsive copolymer with mesoporous silica SBA-15, which was found to be a highly active, eco-friendly, and recyclable catalyst for the selective oxidation of alcohols in water at room temperature. Under optimized reaction conditions, the catalyst was highly effective for the oxidation of various alcohols with substituted alkyl, aryl, and heterocyclic alcohols in the aqueous media using $\mathrm{H}_{2} \mathrm{O}_{2}$ as an oxidant at room temperature. The CuNPs/p(DMAEMA-co-TBA)/TSBA catalyst exhibited some responsive behavior at a certain temperature and $\mathrm{pH}$. The lower critical solution temperature (LCST) became lowered by co-polymerization with a hydrophobic co-monomer (TBA). The LCST of the copolymer was found to be in the range of $25-30{ }^{\circ} \mathrm{C}$. At low temperature and acidic environment, the benzyl alcohol conversion was found to be high. At high temperatures under basic conditions, however, the yield of conversion was less due to the swelling/shrinking of the copolymer coated on mesoporous silica SBA-15. In addition, the catalyst can be reused for at least five reaction cycles without significant loss of catalytic activity. In summary, the hybrid catalyst developed in this study exhibited outstanding alcohol conversion (up to $99 \%$ ) within a short reaction time $(1 \mathrm{~h})$. It should also be noted that only a small amount of catalyst (5 mg) is needed to secure such an efficient catalytic activity.

Supplementary Materials: The following are available online at http:/www.mdpi.com/2079-4991/10/10/2051/ s1, Chemicals, Preparation of SBA-15 and SBA-15 functionalized with TMSPM, Materials characterization, Figure S1: Wide angle XRD (WAXRD) patterns of (a) Copper nanoparticles (CuNPs)/SBA-15 and (b) CuNPs/p(DMAEMA-co-TBA)/TSBA; Figure S2: (a) High-resolution transmission electron microscopy (HRTEM) image of CuNPs/SBA-15 catalyst, and (b) particle size histograms of CuNPs; Figure S3: (a) HRTEM image of CuNPs/p(DMAEMA-co-TBA)/TSBA catalyst. (b) Particle size histograms of CuNPs; Figure S4: $\mathrm{N}_{2}$ adsorption-desorption isotherms and pore size distribution curves (inset) of (a) SBA-15, (b) TSBA, and (c) p(DMAEMA-co-TBA)/TSBA; Figure S5: Particle size of CuNPs/p(DMAEMA-co-TBA)/TSB at different monomer ratios of (a) 9:1, (b) 8:2, (c) 7:3, and (d) 6:4 plotted as a function of temperature at $\mathrm{pH}=7$; Figure $56:{ }^{1} \mathrm{H}$ and ${ }^{13} \mathrm{C}$ NMR spectra of products; Figure S7: WAXRD patterns of CuNPs/p(DMAEMA-co-TBA)/TSBA hybrid catalyst on the oxidation of alcohol after fifth wash; Figure S8: HRTEM image of CuNPs/p(DMAEMA-co-TBA)/TSBA hybrid catalyst on the oxidation of alcohol after fifth wash; Figure S9: FT-IR spectra of CuNPs/p(DMAEMA-co-TBA)/TSBA hybrid catalyst on the oxidation of alcohols at (a) fresh and (b) after fifth wash; Table S1: Brunauer-Emmett-Teller (BET) surface area, pore volume, and pore sizes distribution values of SBA 15, TSBA, and p(DMAEMA-co-TBA)/TSBA; Table S2: Effect of solvent in oxidation of benzyl alcohol; Table S3: Effect of oxidants in oxidation of benzyl alcohol; Table S4: Effect of catalyst amount in oxidation of benzyl alcohol; Table S5: Effect of different catalysts in oxidation of benzyl alcohol; Table S6: Total expense for the synthesis of CuNPs/p(DMAEMA-co-TBA)/TSBA hybrid catalyst; Table S7: Total expense for the synthesis of Copper nanoparticles; Table S8: Total expense for the Synthesis of Iron nanoparticles; Table S9: Total expense for the Synthesis of Iron nanoparticles.

Author Contributions: Conceptualization, C.-S.H.; methodology, A.M.T.; validation, J.P.; formal analysis, A.M.T. and A.M.; investigation, A.M.T.; resources, C.-S.H.; data curation, A.M.T.; writing-original draft preparation, A.M.T.; writing-review and editing, S.N. and C.-S.H.; visualization, J.P.; supervision, C.-S.H.; project administration, C.-S.H.; funding acquisition, C.-S.H. All authors have read and agreed to the published version of the manuscript.

Funding: This research was supported by the National Research Foundation of Korea funded by the Ministry of Science and ICT, Korea (NRF-2017R1A2B3012961; Brain Korea 21 Plus Program (21A2013800002)). 
Conflicts of Interest: The authors declare no conflict of interest.

\section{References}

1. Ragupathi, C.; Vijaya, J.J.; Narayanan, S.; Jesudoss, S.K.; Kennedy, L.J. Highly Selective Oxidation of Benzyl Alcohol to Benzaldehyde with Hydrogen Peroxide by Cobalt Aluminate Catalysis: A Comparison of Conventional and Microwave Methods. Ceram. Int. 2015, 41, 2069-2080. [CrossRef]

2. Xu, J.; Shang, J.K.; Chen, Y.; Wang, Y.; Li, Y.X. Palladium Nanoparticles Supported on Mesoporous Carbon Nitride for Efficiently Selective Oxidation of Benzyl Alcohol with Molecular Oxygen. Appl. Catal. A Gen. 2017, 542, 380-388. [CrossRef]

3. Ali, R.; Nour, K.; Warthan, A.A.; Siddiqui, M.R.H. Selective Oxidation of Benzylic Alcohols Using Copper-Manganese Mixed Oxide Nanoparticles as Catalyst. Arab. J. Chem. 2015, 8, 512-517. [CrossRef]

4. Nozaki, A.; Yasuku, T.; Kuwahara, Y.; Ohmichi, T.; Mori, K.; Nagase, T.; Yasuda, H.Y.; Yamashita, H. Oxidation of Benzyl Alcohol over Nanoporous $\mathrm{Au}-\mathrm{CeO}_{2}$ Catalysts Prepared from Amorphous Alloys and Effect of Alloying Au with Amorphous Alloys. Ind. Eng. Chem. Res. 2018, 57, 5599-5605. [CrossRef]

5. Tian, T.; Liu, Y.; Zhang, X. Bimetallic Synergistic Au/CuO-Hydroxyapatite Catalyst for Aerobic Oxidation of Alcohols. Chin. J. Catal. 2015, 36, 1358-1364. [CrossRef]

6. Williams, R.M.; Medlin, J.W. Benzyl Alcohol Oxidation on Pd(111): Aromatic Binding Effects on Alcohol Reactivity. Langmuir 2014, 30, 4642-4653. [CrossRef]

7. Choudhary, V.R.; Dumbre, D.K.; Bhargava, S.K. Oxidation of Benzyl Alcohol to Benzaldehyde by tert-Butyl Hydroperoxide Over Nanogold Supported S supported on $\mathrm{TiO}_{2}$ and Other Transition and Rare-Earth Metal Oxides. Ind. Eng. Chem. Res. 2009, 48, 9471-9478. [CrossRef]

8. Perez, Y.; Ballesteros, R.; Fajardo, M.; Sierra, I.; Hierro, I.D. Copper-Containing Catalysts for Solvent-Free Selective Oxidation of Benzyl Alcohol. J. Mol. Catal. A Chem. 2012, 352, 45-56. [CrossRef]

9. Mirsafaei, R.; Heravi, M.M.; Hosseinnejad, T.; Ahmadi, S. Copper (II) Nanoparticles: An Efficient and Reusable Catalyst in Green Oxidation of Benzyl Alcohols to Benzaldehydes in Water. Appl. Organometal. Chem. 2016, 30, 823-830. [CrossRef]

10. Feng, X.; Lv, P.; Sun, W.; Han, X.; Gao, L.; Zheng, G. Reduced Graphene Oxide-Supported Cu Nanoparticles for the Selective Oxidation of Benzyl Alcohol to Aldehyde with Molecular Oxygen. Catal. Commun. 2017, 99, 105-109. [CrossRef]

11. Varma, S.V.; Dahiya, R. Copper (II) Nitrate on Clay (claycop)-Hydrogen Peroxide: Selective and Solvent-Free Oxidations Using Microwaves. Tetrahedron Lett. 1998, 39, 1307-1308. [CrossRef]

12. Gawande, M.B.; Goswami, A.; Felpin, F.X.; Asefa, T.; Huang, X.; Silva, R.; Zou, X.; Zboril, R.; Varma, R.S. $\mathrm{Cu}$ and $\mathrm{Cu}-$ Based Nanoparticles: Synthesis and Applications in Catalysis. Chem. Rev. 2016, 116, 3722-3811. [CrossRef] [PubMed]

13. Gracia, D.D.; Ardiles, P.R.; Prashar, S.; Rodriguez, D.A.; Paez, P.L.; Ruiz, S.G. Preparation and Study of the Antibacterial Applications and Oxidative Stress Induction of Copper Maleamate-Functionalized Mesoporous Silica Nanoparticle. Pharmaceutics 2019, 11, 1-18.

14. Saikia, D.; Huang, Y.Y.; Wu, C.E.; Kao, H.M. Size Dependence of Silver Nanoparticles in Carboxylic Acid Functionalized Mesoporous Silica SBA-15 for Catalytic Reduction of 4-Nitrophenol. RSC Adv. 2016, 6, 35167-35176. [CrossRef]

15. Jiang, Y.W.; Chai, K.; Wang, Y.Q.; Zhang, H.D.; Xu, W.; Li, W.; Shi, Y. Mesoporous Silica-Supported $\mathrm{CuCo}_{2} \mathrm{O}_{4}$ Mixed-Metal Oxides for the Aerobic Oxidation of Alcohols. ACS Appl. Nano Mater. 2019, 2, 4435-4442. [CrossRef]

16. Jia, L.; Zhang, S.; Gu, F.; Ping, Y.; Guo, X.; Zhong, Z.; Su, F. Highly Selective Gas-Phase Oxidation of Benzyl Alcohol to Benzaldehyde Over Silver-Containing Hexagonal Mesoporous Silica. Microporous Mesoporous Mater. 2012, 149, 158-165. [CrossRef]

17. Bastakoti, B.P.; Kuila, D.; Salomon, C.; Konarova, M.; Eguchi, M.; Na, J.; Yamauchi, Y. Metal Incorporated Mesoporous Oxides: Synthesis and Applications. J. Hazard. Mater. 2020, 401, 123348-123362. [CrossRef] [PubMed]

18. Tang, Y.J.; Gao, M.R.; Liu, C.H.; Li, S.L.; Jiang, H.L.; Lan, Y.Q.; Han, M.; Yu, S.H. Porous Molybdenum-Based Hybrid Catalysts for Highly Efficient Hydrogen Evolution. Angew. Chem. Int. Ed. 2015, 54, 12928-12932. [CrossRef] 
19. Yano, K. Hollow Metal-Incorporated Monodispersed Mesoporous Silica Spheres. Langmuir 2015, 31, 8774-8779. [CrossRef] [PubMed]

20. Liu, L.; Zou, G.; Yang, B.; Luo, X.; Xu, S. Amine-Functionalized Mesoporous Silica @ Reduced Graphene Sandwichlike Structure Composites for $\mathrm{CO}_{2}$ Adsorption. ACS Appl. Nano Mater. 2018, 1, 4695-4702. [CrossRef]

21. Barczak, M.; Dobrowolski, R.; Borowski, P.; Giannakoudakis, D.A. Pyridine-, Thiol- and Amine-Functionalized Mesoporous Silicas for Adsorptive Removal of Pharmaceuticals. Microporous Mesoporous Mater. 2020, 299, 110132. [CrossRef]

22. Wu, S.H.; Mou, C.Y.; Lin, H.P. Synthesis of Mesoporous Silica Nanoparticles. Chem. Soc. Rev. 2013, 42, 3862-3875. [CrossRef] [PubMed]

23. Galet, V.M.; Esteve, E.P.; Rico, M.R.; Manez, R.M.; Barat, J.M.; Munoz, P.H.; Gavara, R. Anchoring Gated Mesoporous Silica Particles to Ethylene Vinyl Alcohol Films for Smart Packaging Applications. Nanomaterials 2018, 8, 865. [CrossRef] [PubMed]

24. Yu, F.; Tang, X.; Pei, M. Facile synthesis of PDMAEMA-coated Hollow Mesoporous Silica Nanoparticles and their pH-Responsive Controlled Release. Microporous Mesoporous Mater. 2013, 173, 64-69. [CrossRef]

25. Brilmayer, R.; Hess, C.; Brunsen, A.A. Influence of Chain Architecture on Nanopore Accessibility in Polyelectrolyte Block-Co-Oligomer Functionalized Mesopores. Small 2019, 15, 1902710-1902717. [CrossRef] [PubMed]

26. Thomas, A.M.; Mohan, A.; Rout, L.; Nagappan, S.; Park, S.S.; Ha, C.S. Pd Nanoparticle Incorporated Mesoporous Silicas with Excellent Catalytic Activity and Dual Responsivity. Colloid Surf. A Physciochem. Eng. Asp. 2020, 585, 124074-124084. [CrossRef]

27. Lambert, B.D.; Charreyre, M.T.; Chaix, C.; Pichot, C. Poly(N-tert-butyl acrylamide-b-N-acryloylmorpholine) Amphiphilic Block Copolymers Via Polymerization: Synthesis, Purification and Characterization. Polymer 2006, 28, 437-447. [CrossRef]

28. Zhao, D.; Li, X.; Shi, X.; Ye, K.; Liu, W.; Qiu, G.; Lu, X. In Situ Synthesis of Magnetic Poly(N-tert-butyl acrylamide-co-acrylic acid)/ $/ \mathrm{Fe}_{3} \mathrm{O}_{4}$ Nanogels for Magnetic Resonance Imaging. RSC Adv. 2016, 6, 61001-61005. [CrossRef]

29. Raffi, M.; Mehrwan, S.; Bhatti, T.M.; Akhter, J.I.; Hameed, A.; Yawar, W.; Hasan, M.M.U. Investigations into the Antibacterial Behavior of Copper Nanoparticles against Escherichia coli. Ann. Microbiol. 2010, 60, 75-80. [CrossRef]

30. Cruz, P.; Perez, Y.; Hierro, I.D.; Fajardo, M. Copper, Copper Oxide Nanoparticles and Copper Complexes Supported on Mesoporous SBA-15 as Catalysts in the Selective Oxidation of Benzyl Alcohol in Aqueous Phase. Microporous Mesoporous Mater. 2016, 220, 136-147. [CrossRef]

31. Wang, J.; Liu, C.; Hussain, I.; Li, C.; Li, J.; Sun, X.; Shen, J.; Han, H.; Wang, L. Iron-Copper Bimetallic Nanoparticles Supported on Hollow Mesoporous Silica Spheres: The Effect of Fe/Cu Ratio on Heterogeneous Fenton Degradation of a Dye. RSC Adv. 2016, 6, 54623-54635. [CrossRef]

32. Mohan, A.; Rout, L.; Thomas, A.M.; Nagappan, S.; Parambadath, S.; Park, S.S.; Ha, C.S. Silver Nanoparticles Impregnated pH-Responsive Nanohyhybrid System for the Catalytic Reduction of Dyes. Microporous Mesoporous Mater. 2020, 303, 110260-110269. [CrossRef]

33. Liu, F.; Urban, M.W. Dual pH and Temperature Responsiveness of poly(2-(N, N-dimethylamino)ethyl methacrylate-co-n-butyl acrylate)] Colloidal Dispersions and Their Films. Macromolecules 2008, 41, 6531-6539. [CrossRef]

34. Yuan, W.; Zhao, Z.; Yuan, J.; Gu, S.; Zhang, F.; Xie, X.; Ren, J. Synthesis of pH and Temperature-Responsive Chitosan-graft-poly(2-(N,N-dimethylamino)ethyl methacrylate) Copolymer and Gold Nanoparticle Stabilization by its Micelles. Polym. Int. 2011, 60, 194-201. [CrossRef]

35. Ganesamoorthy, S.; Jerome, P.; Shanmugasundaram, K.; Karvembu, R. Highly Efficient Homogeneous and Hetrogenized Ruthenium Catalyst for Transfer Hydrogenation of Carbonyl Compounds. RSC Adv. 2014, 4, 27955-27962. [CrossRef]

36. Babu, S.G.; Priyadarsini, P.A.; Karvembu, R. Copper on Boehmite: A Simple, Selective, Efficient and Reusable Hetrogeneous Catalyst for Oxidation of alcohols with Periodic Acid in Water at Room Temperature. Appl. Catal. A Gen. 2011, 392, 1218-1224. [CrossRef] 
37. Rozner, S.D.; Alsters, P.L.; Neumann, R. Water Soluble and Self-Assembled Polyoxometalate as a Recyclable Catalyst for Oxidation of Alcohols in Water with Hydrogen Peroxide. J. Am. Chem. Soc. 2003, 125, 5280-5281. [CrossRef] [PubMed]

38. Frija, L.M.T.; Alegria, E.C.B.A.; Sutradhar, M.; Cristiano, M.L.S.; Ismael, A.; Kopylovich, M.N.; Pombeiro, A.J.L. Copper(II) and Cobalt(II) Tetrazole-Saccharinate Complexes as Effective Catalysts for Oxidation of Secondary Alcohols. J. Mol. Catal. A Chem. 2016, 425, 283-290. [CrossRef]

39. Ferraz, C.P.; Garcia, M.A.S.; Neto, E.T.; Rossi, L.M. Oxidation of Benzyl Alcohol Catalysed by Gold Catalyzed by Gold Nanoparticles under Alkaline Conditions: Weak vs. Strong Baes. RSC Adv. 2016, 6, 25279-25285. [CrossRef]

40. Xu, S.; Wu, J.; Huang, P.; Lao, C.; Lai, H.; Wang, Y.; Wang, Z.; Zhong, G.; Fu, X.; Peng, F. Selective Catalytic Oxidation of Benzyl Alcohol to Benzaldehyde by Nitrates. Front. Chem. 2020, 8, 151. [CrossRef]

41. Chen, G.J.; Wang, J.S.; Jin, F.Z.; Liu, M.Y.; Zhao, C.W.; Li, Y.A.; Dong, Y.B. Pd@Cu(II)-MOF-Catalysed Aerobic Oxidation of Benzylic Alcohols in Air with High Conversion and Selectivity. Inorg. Chem. 2016, 55, 3058-3064. [CrossRef] [PubMed]

42. Ahmad, J.U.; Raiisanen, M.T.; Leskela, M.; Repo, T. Copper Catalyzed Oxidation of Benzylic Alcohols in Water with $\mathrm{H}_{2} \mathrm{O}_{2}$. Appl. Catal. A Gen. 2012, 411, 180-187. [CrossRef]

43. Meng, C.; Yang, K.; Fu, X.; Yuan, R. Photocatalytic Oxidation of Benzyl Alcohol by Homogeneous $\mathrm{CuCl}_{2}$ /Solvent: A Model System to Explore the Role if Molecular Oxygen. ACS Catal. 2015, 5, 3760-3766. [CrossRef]

44. Ali, R.; Adil, A.F.; Warthan, A.A.; Siddiqui, M.R.H. Identification of Active Phase for Selective Oxidation of Benzyl Alcohol with Moleular Oxygen Catalyzed Copper-Manganese Oxide Nanoparticles. J. Chem. 2012, 2013, 1-9.

45. Lokhande, P.D.; Waghmare, S.R.; Gaikward, H.; Hankare, P.P. Copper Catalyzed Oxidation of Benzyl Alcohol to Benzaldehyde. J. Korean Chem. Soc. 2012, 56, 539-541. [CrossRef]

46. Neves, P.; Valente, A.A.; Lin, Z. Mild Liquid Phase Oxidation of Benzyl Alcohol in the Presence of Microporous Framework Copper Silicates. Chem. Eur. J. 2020, 2020, 1172-1176. [CrossRef]

47. Pina, C.D.; Falletta, E.; Rossi, M. Highly Selective Oxidation of Benzyl Alcohol to Benzaldehyde Catalysed by Bimetallic Gold-Copper Catalyst. J. Catal. 2008, 260, 384-386. [CrossRef]

48. Liu, K.; Quin, T.; Sun, Y.; Hou, C.; Cao, X.; Jiang, S. Synergistic Effect Between Ag and $\mathrm{Mn}_{3} \mathrm{O}_{4}$ in the Gas Phase Oxidation of Alcohols. Catal. Commun. 2018, 113, 15-18. [CrossRef]

49. Lin, Q.; Li, Y.H.; Qi, M.Y.; Li, J.Y.; Tang, Z.R.; Anpo, M.; Yamada, Y.M.A.; Xu, Y.J. Photoredox Dual Reaction for Selective Alcohol Oxidation and Hydrogen Evolution Over Nickel Surface-Modified $\mathrm{ZnIn}_{2} \mathrm{~S}_{4}$. Appl. Catal. B 2020, 271, 118946-118953. [CrossRef]

50. Luo, N.; Wang, M.; Li, H.; Zhang, J.; Liu, H.; Wang, F. Photocatalytic Oxidation-Hydrogenolysis of Lignin $\beta-O-4$ Models Via a Dual Light Wavelength Switching Strategy. ACS Catal. 2016, 6, 7716-7721. [CrossRef]

Publisher's Note: MDPI stays neutral with regard to jurisdictional claims in published maps and institutional affiliations.

(C) 2020 by the authors. Licensee MDPI, Basel, Switzerland. This article is an open access article distributed under the terms and conditions of the Creative Commons Attribution (CC BY) license (http://creativecommons.org/licenses/by/4.0/). 